\title{
NUMERICAL ASPECTS OF THE NONLINEAR SCHRÖDINGER EQUATION IN THE SEMICLASSICAL LIMIT IN A SUPERCRITICAL REGIME*
}

\author{
RÉmi Carles ${ }^{1}$ And Bijan Mohammadi ${ }^{1}$
}

\begin{abstract}
We study numerically the semiclassical limit for the nonlinear Schrödinger equation thanks to a modification of the Madelung transform due to Grenier. This approach allows for the presence of vacuum. Even if the mesh size and the time step do not depend on the Planck constant, we recover the position and current densities in the semiclassical limit, with a numerical rate of convergence in accordance with the theoretical results, before shocks appear in the limiting Euler equation. By using simple projections, the mass and the momentum of the solution are well preserved by the numerical scheme, while the variation of the energy is not negligible numerically. Experiments suggest that beyond the critical time for the Euler equation, Grenier's approach yields smooth but highly oscillatory terms.
\end{abstract}

Mathematics Subject Classification. 35Q55, 65M99, 76A02, 81Q20, 82D50.

Received June 18, 2010. Revised January 8, 2011.

Published online June 13, 2011.

\section{INTRODUCTION}

We consider the cubic nonlinear equation

$$
\mathrm{i} \varepsilon \partial_{t} u^{\varepsilon}+\frac{\varepsilon^{2}}{2} \Delta u^{\varepsilon}=\left|u^{\varepsilon}\right|^{2} u^{\varepsilon}, \quad(t, x) \in \mathbb{R}_{+} \times \mathbb{R}^{d} .
$$

The goal is to compute the solution $u^{\varepsilon}$ in such a way that for $\varepsilon=1$, we solve the nonlinear Schrödinger equation, and in the semiclassical limit $\varepsilon \rightarrow 0$, we retrieve the limit in terms of compressible Euler equation, as recalled below. This equation appears in several contexts in physics. For instance, in the case $\varepsilon=1,(1.1)$ corresponds to an envelope equation in the propagation of lasers, a case where $t$ does not correspond to time, but to the direction of propagation; see e.g. [44] and references therein. The semiclassical regime is present in the modeling of Bose-Einstein condensation, where $\varepsilon$ corresponds to the (rescaled) Planck constant; see e.g. [41] and references therein. A remarkable property in the semiclassical regime is that the limit is expressed in terms of a compressible, isentropic Euler equation.

A popular way to relate the semiclassical limit to fluid dynamics is the use of the Madelung transform [32], which is essentially the polar decomposition: seek the solution to (1.1) of the form

$$
u^{\varepsilon}(t, x)=\sqrt{\rho(t, x)} \mathrm{e}^{\mathrm{i} S(t, x) / \varepsilon}, \quad \rho \geqslant 0, \quad S \in \mathbb{R} .
$$

Keywords and phrases. Nonlinear Schrödinger equation, semiclassical limit, compressible Euler equation, numerical simulation.

* This work was supported by the French ANR project R.A.S. (ANR-08-JCJC-0124-01).

${ }^{1}$ CNRS and Univ. Montpellier 2, Mathématiques, CC 051, 34095 Montpellier, France. remi.carles@math.cnrs.fr 
Plugging this expression into (1.1), and separating real and imaginary parts yields

$$
\left\{\begin{array}{l}
\sqrt{\rho}\left(\partial_{t} S+\frac{1}{2}|\nabla S|^{2}+\rho\right)=\frac{\varepsilon^{2}}{2} \Delta(\sqrt{\rho}), \\
\partial_{t} \sqrt{\rho}+\nabla S \cdot \nabla \sqrt{\rho}+\frac{1}{2} \sqrt{\rho} \Delta S=0 .
\end{array}\right.
$$

Two comments are in order at this stage: the first equation shows that $S$ depends on $\varepsilon$ and the second equation shows that so does $\rho$ in general. We shall underscore this fact by using the notation $\left(S^{\varepsilon}, \rho^{\varepsilon}\right)$. Second, the equation for $S^{\varepsilon}$ can be simplified, provided that $\rho^{\varepsilon}$ has no zero. Introducing the velocity $v^{\varepsilon}=\nabla S^{\varepsilon},(1.2)$ yields the system of quantum hydrodynamics (QHD), see also [21]:

$$
\left\{\begin{array}{l}
\partial_{t} v^{\varepsilon}+v^{\varepsilon} \cdot \nabla v^{\varepsilon}+\nabla \rho^{\varepsilon}=\frac{\varepsilon^{2}}{2} \nabla\left(\frac{\Delta\left(\sqrt{\rho}^{\varepsilon}\right)}{\sqrt{\rho}^{\varepsilon}}\right) \\
\partial_{t} \rho^{\varepsilon}+\operatorname{div}\left(\rho^{\varepsilon} v^{\varepsilon}\right)=0
\end{array}\right.
$$

The term on the right hand side of the equation for $v^{\varepsilon}$ is classically referred to as quantum pressure. In the limit $\varepsilon \rightarrow 0$, this term disappears, and we find the compressible Euler equation:

$$
\left\{\begin{array}{l}
\partial_{t} v+v \cdot \nabla v+\nabla \rho=0 \\
\partial_{t} \rho+\operatorname{div}(\rho v)=0
\end{array}\right.
$$

This approach was used recently to develop an asymptotic preserving scheme for the linear Schrödinger equation $\left(\left|u^{\varepsilon}\right|^{2} u^{\varepsilon}\right.$ is replaced with $\left.V(x) u^{\varepsilon}\right)$, see [17]. The goal of an asymptotic preserving scheme is to have a unified way to compute the solution as $\varepsilon=1$, and to retrieve the limit as $\varepsilon \rightarrow 0$, in such a way that the discretization does not depend on $\varepsilon$; see e.g. [18,28]. As pointed out in [17], the drawback of Madelung transform is that it does not support the presence of vacuum $(\rho=0)$. The point of view that we shall study numerically is due to Grenier [27], and consists in seeking $u^{\varepsilon}$ as

$$
u^{\varepsilon}(t, x)=a^{\varepsilon}(t, x) \mathrm{e}^{\mathrm{i} \phi^{\varepsilon}(t, x) / \varepsilon}, \quad a^{\varepsilon} \in \mathbb{C}, \quad \phi^{\varepsilon} \in \mathbb{R} .
$$

Allowing the amplitude $a^{\varepsilon}$ to be complex-valued introduces an extra degree of freedom, compared to the Madelung transform. The choice of Grenier consists in imposing

$$
\left\{\begin{array}{l}
\partial_{t} \phi^{\varepsilon}+\frac{1}{2}\left|\nabla \phi^{\varepsilon}\right|^{2}+\left|a^{\varepsilon}\right|^{2}=0 \\
\partial_{t} a^{\varepsilon}+\nabla \phi^{\varepsilon} \cdot \nabla a^{\varepsilon}+\frac{1}{2} a^{\varepsilon} \Delta \phi^{\varepsilon}=i \frac{\varepsilon}{2} \Delta a^{\varepsilon} .
\end{array}\right.
$$

In terms of $v^{\varepsilon}=\nabla \phi^{\varepsilon}$, this becomes

$$
\left\{\begin{array}{l}
\partial_{t} v^{\varepsilon}+v^{\varepsilon} \cdot \nabla v^{\varepsilon}+\nabla\left|a^{\varepsilon}\right|^{2}=0 \\
\partial_{t} a^{\varepsilon}+v^{\varepsilon} \cdot \nabla a^{\varepsilon}+\frac{1}{2} a^{\varepsilon} \operatorname{div} v^{\varepsilon}=i \frac{\varepsilon}{2} \Delta a^{\varepsilon} .
\end{array}\right.
$$

In this model, the presence of vacuum $\left(a^{\varepsilon}=0\right)$ is not a problem. We will see that this is so both on a theoretical level and in computational tests. In the limit $\varepsilon \rightarrow 0$, we find formally

$$
\left\{\begin{array}{l}
\partial_{t} v+v \cdot \nabla v+\nabla|a|^{2}=0 \\
\partial_{t} a+v \cdot \nabla a+\frac{1}{2} a \operatorname{div} v=0 .
\end{array}\right.
$$


We check that $(\rho, v)=\left(|a|^{2}, v\right)$ then solves (1.4): (1.8) corresponds to the nonlinear symmetrization of (1.4) (see $[14,33]$ ). Note that for fixed $\varepsilon>0$, equation (1.1) is semilinear (the solution is constructed by a fixed point argument, where the nonlinearity is merely viewed as a perturbation, see e.g. [13]), while the limiting equation (1.4) is quasilinear ("more nonlinear"): this may be viewed as a definition of a supercritical regime in terms of semiclassical limit (see e.g. [11] for a more detailed discussion on this aspect).

In this paper, we have chosen to concentrate on the defocusing cubic nonlinearity, for which the relevance of (1.6) to study the semiclassical limit is proved (see Sect. 2.1). It seems very likely that equivalent numerical results should be available for other nonlinearities, as discussed in Section 2.4, even though in several cases, no theoretical result is available concerning the natural generalization (2.6) of (1.7). Similarly, in the linear setting considered in [17], this modified Madelung transformation should overcome the problem of vacuum pointed out in $[17]$.

We also stress the fact that the convergence of (1.7) towards (1.8) holds so long as no singularity has appeared in the solution of (1.8) (or, equivalently, in (1.4)). Note that except in the very specific case $d=1$ (where the cubic Schrödinger equation is completely integrable), no analytical result seems to be available concerning the asymptotic behavior of $u^{\varepsilon}$ as $\varepsilon \rightarrow 0$ for large time (that is, after a singularity has formed in the solution to the Euler equation). As pointed out in [11], the notion of caustic seems to be different in the case of (1.1), compared to the linear case

$$
\mathrm{i} \varepsilon \partial_{t} \psi^{\varepsilon}+\frac{\varepsilon^{2}}{2} \Delta \psi^{\varepsilon}=V(x) \psi^{\varepsilon},
$$

where several computational results are available past caustics (see e.g. [25,26] and references therein, and Sect. 2.2).

A word of caution is needed concerning the notion of asymptotic preserving scheme, which can be characterized by the following two points. A scheme is asymptotic preserving if:

- It has stability constraints independent of $\varepsilon$ (asymptotic stability).

- It provides a consistent discretization of the limit problem (asymptotic consistency).

We do not claim to present an asymptotic preserving scheme, since we do not establish the above two properties. However, it is possible to solve (1.7) and get estimates in Sobolev spaces uniformly as $\varepsilon \in[0,1]$ (hence $\varepsilon=0$ included); see Section 2.1 for more details and references. Therefore, the system (1.7) seems to be a good candidate to construct an asymptotic preserving scheme in a future research. Note that concerning our numerical scheme, the first condition (asymptotic stability) is examined numerically in Section 4; see Figures 16 and 17.

\subsection{Conserved quantities}

Equation (1.1) enjoys a bi-Hamiltonian structure, and has two quantities which are independent of time:

$$
\begin{aligned}
\text { Mass: } & \frac{\mathrm{d}}{\mathrm{d} t}\left\|u^{\varepsilon}(t)\right\|_{L^{2}\left(\mathbb{R}^{d}\right)}^{2}=0, \\
\text { Energy: } & \frac{\mathrm{d}}{\mathrm{d} t}\left(\left\|\varepsilon \nabla u^{\varepsilon}(t)\right\|_{L^{2}\left(\mathbb{R}^{d}\right)}^{2}+\left\|u^{\varepsilon}(t)\right\|_{L^{4}\left(\mathbb{R}^{d}\right)}^{4}\right)=0 .
\end{aligned}
$$

A third important quantity is conserved, which plays a crucial role, e.g. in the study of finite time blow-up in the case of focusing nonlinearities:

$$
\text { Momentum: } \frac{\mathrm{d}}{\mathrm{d} t} \operatorname{Im} \int_{\mathbb{R}^{d}} \bar{u}^{\varepsilon}(t, x) \varepsilon \nabla u^{\varepsilon}(t, x) \mathrm{d} x=0 .
$$

Plugging the phase/amplitude representation (1.5) into these conservation laws, and passing formally to the limit $\varepsilon \rightarrow 0$, we recover conservation laws associated to the Euler equation (1.4) ([11]):

$$
\frac{\mathrm{d}}{\mathrm{d} t} \int_{\mathbb{R}^{d}} \rho(t, x) \mathrm{d} x=\frac{\mathrm{d}}{\mathrm{d} t} \int_{\mathbb{R}^{d}}\left(\rho|v|^{2}+\rho^{2}\right)(t, x) \mathrm{d} x=\frac{\mathrm{d}}{\mathrm{d} t} \int_{\mathbb{R}^{d}}(\rho v)(t, x) \mathrm{d} x=0 .
$$


Setting $J^{\varepsilon}(t)=x+\mathrm{i} \varepsilon t \nabla$, two other evolution laws are available:

$$
\begin{aligned}
\text { Pseudo-conformal: } & \frac{\mathrm{d}}{\mathrm{d} t}\left(\left\|J^{\varepsilon}(t) u^{\varepsilon}\right\|_{L^{2}\left(\mathbb{R}^{d}\right)}^{2}+t^{2}\left\|u^{\varepsilon}\right\|_{L^{4}\left(\mathbb{R}^{d}\right)}^{4}\right)=t(2-d)\left\|u^{\varepsilon}\right\|_{L^{4}\left(\mathbb{R}^{d}\right)}^{4} . \\
& \frac{\mathrm{d}}{\mathrm{d} t} \operatorname{Re} \int_{\mathbb{R}^{d}} \bar{u}^{\varepsilon}(t, x) J^{\varepsilon}(t) u^{\varepsilon}(t, x) \mathrm{d} x=0 .
\end{aligned}
$$

Passing formally to the limit $\varepsilon \rightarrow 0$, we infer:

$$
\begin{aligned}
& \frac{\mathrm{d}}{\mathrm{d} t} \int_{\mathbb{R}^{d}}\left(|x-t v(t, x)|^{2} \rho(t, x)+t^{2} \rho^{2}(t, x)\right) \mathrm{d} x=(2-d) t \int_{\mathbb{R}^{d}} \rho^{2}(t, x) \mathrm{d} x . \\
& \frac{\mathrm{d}}{\mathrm{d} t} \int_{\mathbb{R}^{d}}(x-t v(t, x)) \rho(t, x) \mathrm{d} x=0 .
\end{aligned}
$$

We discuss this aspect further into details in Section 2.3.

\subsection{Semiclassical limit for NLS: numerical approach}

The most reliable approach so far to study numerically the semiclassical limit for Schrödinger equations seems to be the time-splitting spectral discretization (Lie or Strang splitting, see [8]): one solves alternatively two linear equations,

$$
\mathrm{i} \varepsilon \partial_{t} v^{\varepsilon}+\frac{\varepsilon^{2}}{2} \Delta v^{\varepsilon}=0, \quad \text { and } \quad \mathrm{i} \varepsilon \partial_{t} v^{\varepsilon}=\left|v^{\varepsilon}\right|^{2} v^{\varepsilon} .
$$

Despite the appearance, the second equation is linear, since in view of the gauge invariance, $\partial_{t}\left(\left|v^{\varepsilon}\right|^{2}\right)=0$, so the second equation boils down to $\mathrm{i} \partial_{t} v^{\varepsilon}=\left|v_{I}^{\varepsilon}\right|^{2} v^{\varepsilon}$, where $v_{I}^{\varepsilon}$ denotes the initial value for $v^{\varepsilon}$.

Note that from [34], usual finite-difference schemes for the linear Schrödinger equation may lead to very wrong approximations. Instead, schemes based on the fast Fourier transform (FFT) have been preferred. In [5], it was shown that the time-splitting method, coupled with a trigonometric spectral approximation of the spatial derivative, conserves the total mass, and is gauge-invariant, time-reversible. Moreover, with this approach, the convergence of the scheme in $L^{2}$ is proved, when the nonlinearity in (1.1) is replaced by an external potential. This regime turns out to be far less singular in the limit $\varepsilon \rightarrow 0$ than the nonlinear case of (1.1), as discussed below.

We briefly point out that the numerical study in $[5,6]$ shows that, contrary to the case of the linear Schrödinger equation, to study the semiclassical limit for (1.1) with time-splitting, it is necessary to consider mesh sizes and time steps which are $\mathcal{O}(\varepsilon)$. This is due to the fact that the semiclassical regime is strongly nonlinear (supercritical, in the terminology of [11]): we consider initial data which are $\mathcal{O}(1)$ in $L^{2} \cap L^{\infty}$, and there is no power of $\varepsilon$ in front of the nonlinearity. As a consequence, the semiclassical limit is a "strongly nonlinear" process, since starting with a semilinear Schrödinger equation (for fixed $\varepsilon>0$ ), we come up in the limit $\varepsilon \rightarrow 0$ with a quasilinear equation (the compressible Euler equation).

In [6], it is shown that mesh sizes and time steps must be taken of order $\mathcal{O}(\varepsilon)$, even to recover the behavior of two physically important quantities:

$$
\begin{aligned}
& \text { Position density: } \rho^{\varepsilon}(t, x)=\left|u^{\varepsilon}(t, x)\right|^{2}=\left|a^{\varepsilon}(t, x)\right|^{2} . \\
& \text { Current density: } J^{\varepsilon}(t, x)=\varepsilon \operatorname{Im}\left(\bar{u}^{\varepsilon}(t, x) \nabla u^{\varepsilon}(t, x)\right) .
\end{aligned}
$$

We refer to the numerical results in [6], Example 4.3, which show some important instability in the numerical approximation for (1.1), at least if the time step is large compared to $\varepsilon$ : evidently, the position and current densities cannot be computed correctly if mesh size and time step are independent of $\varepsilon$.

On the contrary, we obtain a good description of $\rho^{\varepsilon}$ and $J^{\varepsilon}$ as $\varepsilon \rightarrow 0$ when studying numerically the system (1.7), even if the time step is independent of $\varepsilon$. Things would probably be similar in the case of the QHD system (1.3), up to the important aspect that the presence of vacuum $\left(\rho^{\varepsilon}=0\right)$ is not allowed in (1.3). 
The idea to explain this difference is the following. To construct directly the wave function $u^{\varepsilon}$ solving (1.1), errors which are large compared to $\varepsilon$ (say of order $\varepsilon^{\alpha}, 0<\alpha<1$ ) lead to instability of order $\mathcal{O}(1)$ on $u^{\varepsilon}$ after a short time (of order $\varepsilon^{1-\alpha}$ ). Among possible sources of errors, we can mention a simple space shift, which is rather likely to occur in numerical studies. This can actually be proved thanks to the approach of Grenier, see [10]. This is due to the strong coupling phase/amplitude in (1.6): a small modification of the amplitude $a^{\varepsilon}$ leads to a modification of the same order for $\phi^{\varepsilon}$. To recover $u^{\varepsilon}$, one has to divide $\phi^{\varepsilon}$ by $\varepsilon$, which is small, so the actual error for $\phi^{\varepsilon}$ may be dramatically increased: this is another way to see that in the semiclassical limit, we consider a supercritical regime.

One can rephrase the above analysis as follows. The semiclassical limit for (1.1) is "strongly nonlinear": as $\varepsilon \rightarrow 0$, we pass from a semilinear equation (for fixed $\varepsilon$ ), to a quasilinear one, the Euler equation (1.4) (in which the nonlinear terms cannot be treated by perturbative methods, see e.g. [45]). As a consequence, the asymptotic behavior of $u^{\varepsilon}$ is very sensitive to small errors [10]. In time splitting methods, one considers the nonlinearity as a perturbation, while this is not sensible in the framework of (1.1), unless a high precision in the space and time steps is demanded. It would be quite different with some positive power - at least 1 - of $\varepsilon$ in front of the nonlinearity; see [11] for theoretical explanations, and [5,6] for numerical illustrations.

If one is interested only in the position and current densities, small errors in (1.7) are not so important, since one never has to divide the phase by $\varepsilon$ (see Sect. 2 for more details). This explains why we can obtain satisfactory results by considering a mesh size $h=\Delta x$ independent of $\varepsilon$, and a time step given by the parabolic scaling, that is, proportional to $h^{2}$.

An extra step in the numerical analysis of nonlinear Schrödinger equations was achieved in [7], where a semi-discrete scheme was introduced, which turns NLS into an almost linear system, in the case $\varepsilon=1$. It is based on a central-difference approximation shifted by a half time-step. For $t_{n}=n \delta t$ and $t_{n+1 / 2}=\left(n+\frac{1}{2}\right) \delta t$, let $u^{n}$ be the approximation at $t=t_{n}$. The scheme is given by

$$
\left\{\begin{aligned}
\mathrm{i} \frac{u^{n+1}-u^{n}}{\delta t}+\frac{1}{2} \Delta\left(\frac{u^{n+1}+u^{n}}{2}\right) & =\psi^{n+1 / 2}\left(\frac{u^{n+1}+u^{n}}{2}\right) \\
\frac{\psi^{n+1 / 2}+\psi^{n-1 / 2}}{2} & =\left|u^{n}\right|^{2}
\end{aligned}\right.
$$

This approach has the advantage of preserving the mass (1.9) and an analogue of the energy (1.10) of the solution [7]:

$$
\int_{\mathbb{R}^{d}}\left|u^{n}\right|^{2}=\int_{\mathbb{R}^{d}}\left|u^{0}\right|^{2}, \quad \text { and } \quad E^{n}=E^{0}, \text { where } \quad E^{n}=\int_{\mathbb{R}^{d}}\left(\left|\nabla u^{n}\right|^{2}+2\left|u^{n}\right|^{2} \psi^{n-1 / 2}-\left(\psi^{n-1 / 2}\right)^{2}\right) .
$$

It does not seem that there is also an analogue of the momentum which is conserved, in the same fashion as (1.11). Note that to adapt this approach numerically in the semiclassical regime, one would also have to consider mesh sizes and time steps which are $\mathcal{O}(\varepsilon)$. Therefore, the approaches in [5-7] do not seem well suited for asymptotic preserving schemes.

In this paper, we present numerical experiments only, and do not claim to justify the approach by numerical analysis arguments. In view of the little knowledge that we have on the behavior of the solution to (1.7) past the critical time for the Euler equation, such a study could reasonably be expected only so long as the solution of the Euler equation remains smooth. Yet, such a study would be an interesting challenge, which we do not address here.

\subsection{Outline of the paper}

In Section 2, we recall the main theoretical results established for the semiclassical analysis of (1.1). The main goal is to state some results which can thereafter be tested numerically to validate the scheme. The numerical implementation is presented in Section 3. Numerical experiments (based on three examples) are discussed in Section 4. We conclude the paper in Section 5. 


\section{The theoretical POINT OF VIEW}

We will always consider initial data of the form

$$
u^{\varepsilon}(0, x)=a_{0}(x) \mathrm{e}^{\mathrm{i} \phi_{0}(x) / \varepsilon}, \quad a_{0} \in \mathbb{C}, \quad \phi_{0} \in \mathbb{R},
$$

where $a_{0}$ and $\phi_{0}$ are smooth, say in $H^{s}\left(\mathbb{R}^{d}\right)$ for all $s$. In that case, (1.6) is supplemented with the Cauchy data

$$
a^{\varepsilon}(0, x)=a_{0}(x) ; \quad \phi^{\varepsilon}(0, x)=\phi_{0}(x) .
$$

This implies that the Cauchy data for (1.7) are

$$
a^{\varepsilon}(0, x)=a_{0}(x) ; \quad v^{\varepsilon}(0, x)=\nabla \phi_{0}(x) .
$$

\subsection{Known results}

A second advantage of the system (1.7) over (1.3), besides the role of vacuum, is that it already has the form of an hyperbolic symmetric system. Separate real and imaginary parts of $a^{\varepsilon}, a^{\varepsilon}=a_{1}^{\varepsilon}+\mathrm{i} a_{2}^{\varepsilon},(1.7)$ takes the form

$$
\begin{gathered}
\partial_{t} \mathbf{u}^{\varepsilon}+\sum_{j=1}^{n} A_{j}\left(\mathbf{u}^{\varepsilon}\right) \partial_{j} \mathbf{u}^{\varepsilon}=\frac{\varepsilon}{2} L \mathbf{u}^{\varepsilon}, \text { with } \quad \mathbf{u}^{\varepsilon}=\left(\begin{array}{c}
a_{1}^{\varepsilon} \\
a_{2}^{\varepsilon} \\
v_{1}^{\varepsilon} \\
\vdots \\
v_{d}^{\varepsilon}
\end{array}\right), \\
L=\left(\begin{array}{ccccc}
0 & -\Delta & 0 & \ldots & 0 \\
\Delta & 0 & 0 & \ldots & 0 \\
0 & 0 & & 0_{d \times d}
\end{array}\right) \text {, and } A(\mathbf{u}, \xi)=\sum_{j=1}^{d} A_{j}(\mathbf{u}) \xi_{j}=\left(\begin{array}{ccc}
v \cdot \xi & 0 & \frac{a_{1}}{2} \xi \\
0 & v \cdot \xi & \frac{a_{2}}{2} \xi \xi \\
2 a_{1} \xi & 2 a_{2} \xi & v \cdot \xi I_{d}
\end{array}\right) .
\end{gathered}
$$

The matrix $A$ is symmetrized by a constant diagonal matrix $S$ such that $S L=L$. We note that $L$ is skewsymmetric, so it plays no role in energy estimates in Sobolev spaces $H^{s}\left(\mathbb{R}^{d}\right)$. The main results in [27] can be summarized as follows:

Theorem 2.1 (from [27]). Let $d \geqslant 1, s>4+d / 2$, and $a_{0}, \nabla \phi_{0} \in H^{s}\left(\mathbb{R}^{d}\right)$.

1. There exist $T>0$ and a unique solution $(\rho, v) \in C\left([0, T] ; H^{s}\left(\mathbb{R}^{d}\right)\right)^{2}$ to (1.4) such that $\rho(0, x)=\left|a_{0}(x)\right|^{2}$ and $v(0, x)=\nabla \phi_{0}(x)$.

2. For the same $T,(1.7)$ has a unique solution $\left(a^{\varepsilon}, v^{\varepsilon}\right) \in C\left([0, T] ; H^{s-2}\left(\mathbb{R}^{d}\right)\right)^{2}$ such that $a^{\varepsilon}(0, x)=a_{0}(x)$ and $v^{\varepsilon}(0, x)=\nabla \phi_{0}(x)$.

$2^{\prime}$. For the same $T,(1.8)$ has a unique solution $(a, v) \in C\left([0, T] ; H^{s}\left(\mathbb{R}^{d}\right)\right)^{2}$ such that $a(0, x)=a_{0}(x)$ and $v(0, x)=\nabla \phi_{0}(x)$.

3. As $\varepsilon \rightarrow 0$, we have: $\left\|a^{\varepsilon}-a\right\|_{L^{\infty}\left([0, T] ; H^{s-2}\right)}+\left\|v^{\varepsilon}-v\right\|_{L^{\infty}\left([0, T] ; H^{s-2}\right)}=\mathcal{O}(\varepsilon)$.

Remark 2.2 (periodic case). The same result holds in the periodic setting ( $x \in \mathbb{T}^{d}$ instead of $x \in \mathbb{R}^{d}$ ), with exactly the same proof.

Once $v^{\varepsilon}$ is constructed, there are at least two ways to get back to $\phi^{\varepsilon}$. Either argue that $v^{\varepsilon}$ remains irrotational, or simply define $\phi^{\varepsilon}$ as

$$
\phi^{\varepsilon}(t)=\phi_{0}-\int_{0}^{t}\left(\frac{1}{2}\left|v^{\varepsilon}(s)\right|^{2}+\nabla\left|a^{\varepsilon}(s)\right|^{2}\right) \mathrm{d} s,
$$

and check that $\partial_{t}\left(v^{\varepsilon}-\nabla \phi^{\varepsilon}\right)=\partial_{t} v^{\varepsilon}-\nabla \partial_{t} \phi^{\varepsilon}=0$. So for $t \in[0, T]$, that is so long as the solution to the Euler equation (1.4) remains smooth, the solution to (1.1) with initial data $u_{\mid t=0}^{\varepsilon}=a_{0} \mathrm{e}^{\mathrm{i} \phi_{0} / \varepsilon}$ is given by $u^{\varepsilon}=a^{\varepsilon} \mathrm{e}^{\mathrm{i} \phi^{\varepsilon} / \varepsilon}$.

Note that even if $\phi_{0}=0, \phi^{\varepsilon}$ (as well as $v^{\varepsilon}$ and $v$ ) must not be expected to be zero (nor even small), because of the strong coupling in (1.6). Typically, if $\phi_{0}=0,(1.6)$ yields $\partial_{t} \phi_{\mid t=0}^{\varepsilon}=-\left|a_{0}\right|^{2} \neq 0$. 
In addition, in the limit $\varepsilon \rightarrow 0$, we recover the main two quadratic observables:

$$
\begin{aligned}
& \rho^{\varepsilon}=\left|a^{\varepsilon}\right|^{2} \underset{\varepsilon \rightarrow 0}{\longrightarrow}|a|^{2}=\rho \text { in } L^{\infty}\left([0, T] ; L^{1}\left(\mathbb{R}^{d}\right)\right), \\
& J^{\varepsilon}=\operatorname{Im}\left(\varepsilon \bar{u}^{\varepsilon} \nabla u^{\varepsilon}\right)=\left|a^{\varepsilon}\right|^{2} v^{\varepsilon}+\varepsilon \operatorname{Im}\left(\bar{a}^{\varepsilon} \nabla a^{\varepsilon}\right) \underset{\varepsilon \rightarrow 0}{\longrightarrow}|a|^{2} v=J \text { in } L^{\infty}\left([0, T] ; L^{1}\left(\mathbb{R}^{d}\right)\right) .
\end{aligned}
$$

We have more precisely:

$$
\left\|\rho^{\varepsilon}-\rho\right\|_{L^{\infty}\left([0, T] ; L^{1} \cap L^{\infty}\right)}+\left\|J^{\varepsilon}-J\right\|_{L^{\infty}\left([0, T] ; L^{1} \cap L^{\infty}\right)}=\mathcal{O}(\varepsilon) .
$$

We can also prove the convergence of the wave function ([27]). In the particular case which we consider where the initial amplitude $a^{\varepsilon}(0, x)$ does not depend on $\varepsilon$, we have (with an obvious definition for $\phi$ ):

$$
\left\|u^{\varepsilon}-a \mathrm{e}^{\mathrm{i} \phi / \varepsilon}\right\|_{L^{\infty}\left([0, T] ; L^{2} \cap L^{\infty}\right)}=\mathcal{O}(\varepsilon) .
$$

In general, a modulation of $a$ must be taken into account to have such an approximation of the wave function ([11]): $u^{\varepsilon} \approx a \mathrm{e}^{\mathrm{i} \phi^{(1)}} \mathrm{e}^{\mathrm{i} \phi / \varepsilon}$. In the framework of this paper, we have $\phi^{(1)}=0$ (see [11], Sect. 4.2).

\subsection{An open question}

As pointed out in the introduction, no analytical result seems to be available concerning the semiclassical limit of (1.1) when the solution of the Euler equation (1.4) has become singular. Theorem 2.1 gives a rather complete picture for the asymptotic behavior of $u^{\varepsilon}$ for $t \in[0, T]$, that it before the solution to (1.4) becomes singular. Note that if for instance $a_{0}$ and $\phi_{0}$ are compactly supported, then no matter how small they are, $(a, v)$ develops a singularity in finite time $([14,33,47])$. On the other hand, for fixed $\varepsilon>0$, we know that the solution to (1.1) with initial data $u_{\mid t=0}^{\varepsilon}=a_{0} \mathrm{e}^{\mathrm{i} \phi_{0} / \varepsilon} \in H^{s}\left(\mathbb{R}^{d}\right), s \geqslant 1$, is global in time with the same regularity, at least if $d \leqslant 4: u^{\varepsilon} \in C\left(\left[0, \infty\left[; H^{s}\left(\mathbb{R}^{d}\right)\right)\right.\right.$. See [24] (or [13]) for the case $d \leqslant 3$, and [42] for the case $d=4$ (which is energy-critical).

A natural question is then: what happens to $u^{\varepsilon}$ as the solution to the Euler equation (1.4) becomes singular? In the linear setting,

$$
\mathrm{i} \varepsilon \partial_{t} u_{\operatorname{lin}}^{\varepsilon}+\frac{\varepsilon^{2}}{2} \Delta u_{\operatorname{lin}}^{\varepsilon}=0 ; \quad u_{\operatorname{lin} \mid t=0}^{\varepsilon}=a_{0} \mathrm{e}^{\mathrm{i} \phi_{0} / \varepsilon}
$$

the question is rather well understood: when the solution to the corresponding Burger's equation (for the phase) becomes singular, a caustic is formed, which is a set in $(t, x)$-space (see e.g. $[19,36])$. Near the caustic, the amplitude of $u_{\text {lin }}^{\varepsilon}$ is amplified, like a negative power of $\varepsilon$. For instance, if $\phi_{0}(x)=-|x|^{2} / 2$, then

$$
u_{\operatorname{lin}}^{\varepsilon}(t, x) \underset{\varepsilon \rightarrow 0}{\sim}\left\{\begin{array}{l}
\frac{1}{(1-t)^{d / 2}} a_{0}\left(\frac{x}{1-t}\right) \mathrm{e}^{\mathrm{i}|x|^{2} /(2 \varepsilon(t-1))} \text { if } t<1, \\
\frac{1}{\varepsilon^{d / 2}} \widehat{a}_{0}\left(\frac{x}{\varepsilon}\right) \text { if } t=1,
\end{array}\right.
$$

where $\widehat{a}_{0}$ denotes the Fourier transform of $a_{0}$; see [11] for several developments around this example, and [12] for corresponding numerical experiments. Such a concentration is ruled out in the case of (1.1), since the conservation of the energy (1.10) yields the uniform bound

$$
\left.\left.\left\|u^{\varepsilon}(t)\right\|_{L^{4}\left(\mathbb{R}^{d}\right)} \leqslant C \text { independent of } \varepsilon \in\right] 0,1\right] \text { and } t \in \mathbb{R} .
$$

We remark that multiplying each equation in (1.7), derivatives become exactly $\varepsilon$-derivatives: every time a term is differentiated, it is multiplied by $\varepsilon$. This is consistent with the possibility that $a^{\varepsilon}$ and $v^{\varepsilon}$ become oscillatory past the critical time for the Euler equation (with wavelength of order $\varepsilon$ or more). The numerical experiments we present below suggest that this is indeed the case. We insist on the fact that no result is available, though, on 
global existence aspects for (1.7): the solution may be globally smooth (and $\varepsilon$-oscillatory, in the sense of [23]), but it may blow up in finite time.

Note however that the approach we present here is no longer expected to be weakly asymptotic preserving (in the sense given in the introduction) beyond the breakup time for the Euler equation. The presence of rapid oscillations is a possible explanation, and we then recover the problem pointed out in [5] for pre-breakup times: rapid oscillations can be resolved only if time step and mesh sizes are comparable to the (small) wavelength of the wave. Finally, we point out that even in the linear case (see e.g. the above example), one cannot expect an asymptotic preserving approach to solve (2.5) after a caustic has formed: near the caustic, small spatial scales must be taken into account. In the above example, the wave function is concentrated at scale $\varepsilon$. A possibility to get an aymptotically preserving approach in the linear case would be the use of Lagrangian integrals [19]; see [11] for an extension in a very specific nonlinear setting. Note however that the definition of the Lagrangian integral depends on the initial phase, so this approach is more delicate to implement numerically. The $K$-branch approach would lead to similar requirements; see $[9,25,26]$.

\subsection{Conserved quantities}

In the one-dimensional case $d=1$, the cubic nonlinear Schrödinger equation (1.1) has infinitely many conserved quantities [49] (it is completely integrable, see [48]). We shall not emphasize this particular case in this paper, and rather consider the case of a cubic nonlinearity in arbitrary dimension. Numerical experiments are presented in the two-dimensional case $d=2$.

In this general case, we retain the three standard conservations: mass (1.9), momentum (1.11), and energy (1.10). Writing the solution to (1.1) as $u^{\varepsilon}=a^{\varepsilon} \mathrm{e}^{\mathrm{i} \phi^{\varepsilon} / \varepsilon}$, we infer three corresponding conversation laws for the solution to (1.7):

Proposition 2.3. Let $d \geqslant 1$ and $\left(a^{\varepsilon}, v^{\varepsilon}\right) \in C\left([0, T] ; H^{1} \cap L^{\infty}\left(\mathbb{R}^{d}\right)\right)^{2}$ solve (1.7). The following three quantities do not depend on time:

(1) The $L^{2}$-norm of $a^{\varepsilon}: \frac{\mathrm{d}}{\mathrm{d} t}\left\|a^{\varepsilon}(t)\right\|_{L^{2}\left(\mathbb{R}^{d}\right)}^{2}=0$.

(2) The momentum: $\frac{\mathrm{d}}{\mathrm{d} t} \int_{\mathbb{R}^{d}}\left(\left|a^{\varepsilon}(t, x)\right|^{2} v^{\varepsilon}(t, x)+\varepsilon \operatorname{Im}\left(\bar{a}^{\varepsilon}(t, x) \nabla a^{\varepsilon}(t, x)\right)\right) \mathrm{d} x=0$.

(3) The energy: if $v_{\mid t=0}^{\varepsilon}$ is irrotational, $\nabla \wedge v_{\mid t=0}^{\varepsilon}=0$, then

$$
\frac{\mathrm{d}}{\mathrm{d} t} \int_{\mathbb{R}^{d}}\left(\left|\varepsilon \nabla a^{\varepsilon}(t, x)+\mathrm{i} a^{\varepsilon}(t, x) v^{\varepsilon}(t, x)\right|^{2}+\left|a^{\varepsilon}(t, x)\right|^{4}\right) \mathrm{d} x=0 .
$$

Sketch of proof. This result can be proved by using the standard regularizing procedure and suitable multipliers. We shall just indicate the formal procedure.

The conservation of mass is proved by multiplying the second equation in (1.7) by $\bar{a}^{\varepsilon}$, integrating in space, and taking the real value.

The conservation of the momentum is obtained as follows. Multiply the equation for $v^{\varepsilon}$ by $\frac{1}{2}\left|a^{\varepsilon}\right|^{2}$, and integrate in space. Multiply the equation for $a^{\varepsilon}$ by $\mathrm{i} \varepsilon \nabla \bar{a}^{\varepsilon}+\bar{a}^{\varepsilon} v^{\varepsilon}$, integrate in space and consider the real value. Summing these two relations yields the conservation of the momentum.

For the energy, the procedure is similar. Note that

$$
\partial_{t} v^{\varepsilon}=-\nabla\left(\frac{\left|v^{\varepsilon}\right|^{2}}{2}+\left|a^{\varepsilon}\right|^{2}\right), \quad \text { hence } \partial_{t}\left(\nabla \wedge v^{\varepsilon}\right)=0
$$

Therefore, if $\nabla \wedge v_{\mid t=0}^{\varepsilon}=0$, then we can find $\phi^{\varepsilon}$ such that $\left(\phi^{\varepsilon}, a^{\varepsilon}\right)$ solves (1.6). Multiply the equation in $\phi^{\varepsilon}$ by $-\frac{1}{2} \partial_{t}\left|a^{\varepsilon}\right|^{2}$, the equation for $a^{\varepsilon}$ by $\mathrm{i} \varepsilon \partial_{t} \bar{a}^{\varepsilon}+\bar{a}^{\varepsilon} \partial_{t} \phi^{\varepsilon}$. Sum up the two equations, integrate in space, and take the real part. 


\subsection{About other nonlinearities}

Equation (1.1) is the defocusing cubic nonlinear Schrödinger equation. Other nonlinearities are physically relevant too: focusing or defocusing nonlinearities are considered, as well as other powers, in the context of laser Physics (see e.g. [44]) or in the context of Bose-Einstein Condensation (see e.g. [16,29]), for instance.

The (short time) semiclassical limit for nonlinear Schrödinger equations has been studied rigorously for other nonlinearities. Typically, for defocusing nonlinearities

$$
\mathrm{i} \varepsilon \partial_{t} u^{\varepsilon}+\frac{\varepsilon^{2}}{2} \Delta u^{\varepsilon}=\left|u^{\varepsilon}\right|^{2 \sigma} u^{\varepsilon}, \quad \sigma \in \mathbb{N}
$$

a result similar to Theorem 2.1 is available; see $[3,15]$. However, the analysis does not rely on an extension of (1.6) where $\left|a^{\varepsilon}\right|^{2}$ would be replaced with $\left|a^{\varepsilon}\right|^{2 \sigma}$ : for $\varepsilon=0$ (corresponding to the limiting Euler equation in the case $\sigma \in \mathbb{N}$ too), one uses a nonlinear symmetrizer (the "good" unknown is $\left(\nabla \phi, a^{\sigma}\right)$ ), and for $\varepsilon>0$, this change of variable affects the skew-symmetric term i $\varepsilon \Delta a^{\varepsilon}$ in such a way that apparently the analysis of [27] cannot be directly adapted.

For focusing nonlinearities, typically

$$
\mathrm{i} \varepsilon \partial_{t} u^{\varepsilon}+\frac{\varepsilon^{2}}{2} \Delta u^{\varepsilon}=-\left|u^{\varepsilon}\right|^{2 \sigma} u^{\varepsilon}, \quad \sigma \in \mathbb{N}
$$

the limiting equation in the system analogous to (1.4) is elliptic (as opposed the hyperbolic system (1.4)). It turns out that in this case, the "elliptic Euler system" is ill-posed in Sobolev spaces ([37]): working with analytic regularity becomes necessary [37], and sufficient [22,46] in order to justify the semiclassical analysis.

An hybrid nonlinearity (neither focusing, nor defocusing) also plays a role in physical models: the cubicquintic nonlinearity,

$$
\mathrm{i} \varepsilon \partial_{t} u^{\varepsilon}+\frac{\varepsilon^{2}}{2} \Delta u^{\varepsilon}=\left|u^{\varepsilon}\right|^{4} u^{\varepsilon}+\lambda\left|u^{\varepsilon}\right|^{2} u^{\varepsilon}
$$

with $\lambda \in \mathbb{R}$ possibly negative. This model is mostly used as an envelope equation in optics, is also considered in BEC for alkalimetal gases (see e.g. $[1,20,38]$ ), in which case $\lambda<0$. The cubic term corresponds to a negative scattering length, and the quintic term to a repulsive three-body elastic interaction. Justifying the semiclassical analysis was achieved in [4] by a slight modification of the approach of [27] (in a different functional framework).

To rephrase the above discussion, the approach in [27] to study the semiclassical limit for

$$
\mathrm{i} \varepsilon \partial_{t} u^{\varepsilon}+\frac{\varepsilon^{2}}{2} \Delta u^{\varepsilon}=f\left(\left|u^{\varepsilon}\right|^{2}\right) u^{\varepsilon}
$$

relies on the assumption $f^{\prime}>0$. However, the analysis has been carried out in several other situations, without considering the natural generalization of (1.7),

$$
\left\{\begin{array}{l}
\partial_{t} v^{\varepsilon}+v^{\varepsilon} \cdot \nabla v^{\varepsilon}+\nabla f\left(\left|a^{\varepsilon}\right|^{2}\right)=0 \\
\partial_{t} a^{\varepsilon}+v^{\varepsilon} \cdot \nabla a^{\varepsilon}+\frac{1}{2} a^{\varepsilon} \operatorname{div} v^{\varepsilon}=\mathrm{i} \frac{\varepsilon}{2} \Delta a^{\varepsilon}
\end{array}\right.
$$

It seems reasonable to believe that even though no rigorous study for this system is available in general (for $\varepsilon>0$ ), this system can be used for numerical simulations.

Finally, the approach of [27] was generalized to the case where an external potential is introduced (which may model a confining trap in the framework of Bose-Einstein Condensation), see [11], and to the case of Schrödinger-Poisson system $[2,30,31,35]$. 


\section{NumericAl imPLEMENTATION}

One expects the oscillatory nature of the solutions to be difficult to capture numerically. We would like to use a stable numerical scheme with the time step independent of $\varepsilon$ but function of $h$. The scheme solves system (1.6) on coarser meshes than what necessary to capture all wavelengths. Therefore, the solution has inevitably error in it. Still we give a great deal of effort on conservation issues for the density, energy and momentum. The time step and mesh size being both independent of $\varepsilon$, one can tackle very small $\varepsilon$ values and the scheme also works for $\varepsilon=\mathcal{O}(1)$. Obviously, if all scales are aimed at being captured, then the space grid size need be of order of $\varepsilon$ or less and so the time step.

Present results show that the scheme being conservative and stable macroscopic quantities remain observable even when the spatial-temporal oscillations are not fully resolved numerically because the mesh is not enough fine to capture wavelengths below $h$. Typically, one uses $h=0.01$ for all $\varepsilon$ in a square domain of side one.

In our approach, conservation is ensured by projection steps to guarantee a correct behavior for total position, energy and current (momentum) densities. The aim is also to show that basic numerical methods [39,43] can be used which permits the adaptation of generic PDE solvers. We also point out that we have privileged projections which are rather cheap computationally, since they are obtained by a simple rescaling.

The implementation has been done in two dimensions in space but extension to third dimension does not appear being a difficulty. Periodic boundary conditions and initial data with compact support have been considered.

Let us start with system (1.7) which we rewrite as:

$$
\begin{gathered}
\partial_{t} U^{\varepsilon}+F\left(U^{\varepsilon}\right)=0, \\
U^{\varepsilon}(x \in \Omega, t=0)=U_{0}(x), \quad U^{\varepsilon}(\partial \Omega, t)=\text { periodic. }
\end{gathered}
$$

where $U^{\varepsilon}=\left(a^{\varepsilon}, v^{\varepsilon}\right) . \quad \Omega_{h}$ is a discrete two dimensional square domain of side $L$. $U_{0}(x)$ is a regular initial condition. For all the simulations presented in this paper we consider

$$
U_{0}(x)=\left(a_{0}(x), \alpha f(x), \alpha g(x)\right)^{t},
$$

with $a_{0}(x)$ a complex function independent of $\varepsilon$ with compact support. $\alpha$ is real and $f$ and $g$ are real functions with compact support. The initial pattern is therefore periodic of period $L$ in both space directions. Together with the periodicity, oscillations in space can be introduced through $a_{0}, f$ and $g$. Below we show numerical results with two values of $\alpha$.

We consider second order finite difference discretizations of partial differential operators. But, the periodic boundary conditions permit the implementation of high order spatial discretizations as well as spectral methods. One notices that despite the presence of first order space derivatives, no numerical viscosity is necessary to stabilize the system both in the hydrodynamic limit and for $\varepsilon \neq 0$. We therefore keep the numerical viscosity to zero for all simulations which means no upwinding has been used. This leads to a consistent scheme with truncation error in $h^{2}$ :

$$
F\left(U^{\varepsilon}\right)=F_{h}\left(U^{\varepsilon}\right)+\mathcal{O}\left(h^{2}\right) .
$$

We consider a simple first order explicit time integration scheme:

$$
\begin{gathered}
\frac{1}{k}\left(U_{h, n+1 / 2}^{\varepsilon}-U_{h, n}^{\varepsilon}\right)+F_{h}\left(U_{h, n}^{\varepsilon}\right)=0, \\
U_{h, 0}^{\varepsilon}=U_{0}\left(x_{h}\right), \quad U_{h, n+1 / 2}^{\varepsilon}\left(\partial \Omega_{h}\right)=\text { periodic. }
\end{gathered}
$$

$n+1 / 2$ denotes an intermediate state, before projection, where conservation is not guaranteed for mass and momentum. It is interesting that the approach appears stable even for explicit time integration. With a first order scheme in time, and a time step in $h^{2}$, the time integration error will be comparable to the truncation error in space. 
Once $U_{h, n+1 / 2}^{\varepsilon}$ is computed, one needs to project it over the admissible space to get $U_{h, n+1}^{\varepsilon}$ based on enforcing mass, energy and momentum conservation constraints (see Prop. 2.3):

$$
J_{1,2,3}\left(U_{h, n+1 / 2}^{\varepsilon}, U_{h, 0}^{\varepsilon}\right)=\frac{I_{1,2,3}\left(U_{h, n+1 / 2}^{\varepsilon}\right)}{I_{1,2,3}\left(U_{h, 0}^{\varepsilon}\right)}=1,
$$

where

$$
\begin{aligned}
& I_{1}\left(U_{h, n+1 / 2}^{\varepsilon}\right)=\int_{\Omega_{h}}\left|a_{h, n+1 / 2}^{\varepsilon}\right|^{2} \mathrm{~d} x, \\
& I_{2}\left(U_{h, n+1 / 2}^{\varepsilon}\right)=\int_{\Omega_{h}}\left(\left|a_{h, n+1 / 2}^{\varepsilon}\right|^{4}+\left|\varepsilon \nabla_{h} a_{h, n+1 / 2}^{\varepsilon}+\mathrm{i} a_{h, n+1 / 2}^{\varepsilon} v_{h, n+1 / 2}^{\varepsilon}\right|^{2}\right) \mathrm{d} x, \\
& I_{3}\left(U_{h, n+1 / 2}^{\varepsilon}\right)=\int_{\Omega_{h}}\left(\left|a_{h, n+1 / 2}^{\varepsilon}\right|^{2} v_{h, n+1 / 2}^{\varepsilon}+\varepsilon \operatorname{Im}\left(\bar{a}_{h, n+1 / 2}^{\varepsilon} \nabla a_{h, n+1 / 2}^{\varepsilon}\right)\right) \mathrm{d} x .
\end{aligned}
$$

$J_{3}$ is a vector of the size $d$ of the space dimension. This problem is overdetermined with essentially two variables $\left(a^{\varepsilon}\right.$ and $\left.v^{\varepsilon}\right)$. This overdetermination is maybe one reason why no numerical scheme is available for these equations verifying all conservation constraints. With $a^{\varepsilon}$ complex, there are as many variables as constraints. Still we did not manage to enforce at the same time the mass $J_{1}$ and energy $J_{2}$ constraints, and only managed to enforce $J_{1}$ and $J_{3}$.

$J_{1}$ can be easily enforced in $a_{h, n+1}^{\varepsilon}$ by simply defining:

$$
a_{h, n+1}^{\varepsilon}=a_{h, n+1 / 2}^{\varepsilon}\left(\frac{I_{1}\left(U_{h, 0}^{\varepsilon}\right)}{I_{1}\left(U_{h, n+1 / 2}^{\varepsilon}\right)}\right)^{1 / 2} .
$$

The projection aims at looking for a particular equilibrium for the constraints after a splitting of the variables. The above scaling suggests an a priori but natural splitting of the variables to be modified by each constraint. More precisely, $J_{1}$ defines the corrections for $a_{h, n+1 / 2}^{\varepsilon}$ and the vector $J_{3}$ the ones for the components $\left(v_{h, n+1 / 2}^{\varepsilon}\right)_{j}$ of the velocity through:

$$
\left(\tilde{I}_{3}\right)^{j}=\int_{\Omega_{h}}\left(\left|a_{h, n+1}^{\varepsilon}\right|^{2}\left(v_{h, n+1 / 2}^{\varepsilon}\right)_{j}+\varepsilon \operatorname{Im}\left(\bar{a}_{h, n+1}^{\varepsilon} \partial_{j} a_{h, n+1}^{\varepsilon}\right)\right) \mathrm{d} x, \quad j=1, \ldots, d .
$$

Because we are looking for a cheap projection based on scaling, we adopt the following corrections for each component of $v_{h, n+1 / 2}^{\varepsilon}$ :

$$
\left(v_{h, n+1}^{\varepsilon}\right)_{j}=\left(v_{h, n+1 / 2}^{\varepsilon}\right)_{j}\left(\frac{I_{3}^{j}\left(U_{h, 0}^{\varepsilon}\right)}{\tilde{I}_{3}^{j}}\right), \quad j=1, \ldots, d .
$$

Through the numerical examples below we see that these scalings are efficient in conserving mass and current densities.

Remark 3.1. An alternative but more costly approach could be based on minimization under equality constraints. Indeed, at each time iteration, we would like to minimize the following cost function:

$$
R\left(U_{h, n+1}^{\varepsilon}\right)=\frac{1}{2}\left\|U_{h, n+1}^{\varepsilon}-U_{h, n}^{\varepsilon}+k\left(F_{h}\left(U_{h, n}^{\varepsilon}\right)+G_{\partial \Omega_{h}}\left(U_{h, n}^{\varepsilon}\right)\right)\right\|_{0}^{2}
$$

where $U_{h, n+1}^{\varepsilon}$ is the control parameter, $G_{\partial \Omega_{h}}$ a periodic boundary condition operator over the boundary of the domain and $\|\cdot\|_{0}$ the discrete $L^{2}$ norm. Without constraint, the first order optimality condition would realize 

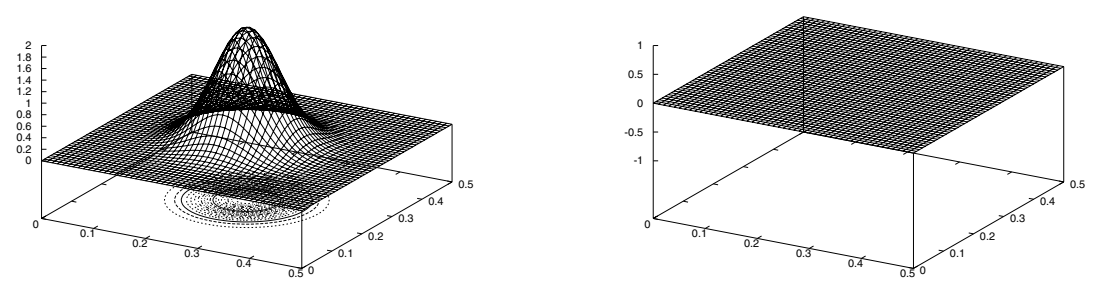

FIGURE 1. Initial position (left) and norm of the current density vector (right) with $\alpha=10^{-10}$ (data considered in Sect. 4.1).

$U_{h, n+1}^{\varepsilon}=U_{h, n}^{\varepsilon}-k\left(F_{h}\left(U_{h, n}^{\varepsilon}\right)+G_{\partial \Omega_{h}}\left(U_{h, n}^{\varepsilon}\right)\right)$. But, this minimization should take place under the constraints $I_{1}=I_{10}, I_{2}=I_{20}, I_{3}=I_{30}$. A Lagrangian can then be defined as

$$
L\left(U_{h, n+1}^{\varepsilon}, \lambda_{1}, \lambda_{2}, \lambda_{3}\right)=R\left(U_{h, n+1}^{\varepsilon}\right)+\sum_{j=1}^{3} \lambda_{j}\left(I_{j}-I_{j 0}\right), \quad \lambda_{j} \in \mathbb{R} .
$$

$U_{h, n+1}^{\varepsilon}$ is solution of an optimality condition for $L$. This could be solved using a dual approach with the Uzawa algorithm, for instance, to find the saddle point solution of the Lagrangian. This is obviously much more costly than the simple projection we used here: in the present case, we assess the cost function and the constraints. In Uzawa algorithm, at each time iteration, a min-max problem must be solved, which consists of two coupled minimization problems: written in a condensed form, $U^{(k)}$ minimizes $L\left(U, \lambda^{(k)}\right)$, while $\lambda^{(k)}$ maximizes $L\left(U^{(k)}, \lambda\right)$ (see e.g. [39] or [40] for more details). This remark suggests however that our approach provides an alternative for the approximate solution of such saddle-point problems by taking a single step in some convenient direction instead of looking for the minimum of the first and the maximum of the second optimization problems of the Uzawa algorithm.

\section{NUMERICAL EXPERIMENTS}

We show the application of our projection schemes for several initial conditions. In the first case the current density is nearly zero and not in the second. A third case shows the robustness of the approach with initial vanishing $a^{\varepsilon}$. We show the impact of the projection on the conservation of mass, energy and momentum through $J_{1}, J_{2}$ and $J_{3}$. We will see that mass and energy cannot be both conserved at the same time.

Throughout this section, all the tests are made on a $50 \times 50$ grid (the mesh size is thus $h=1 / 100$, since we take $L=0.5$ ), and the time step is equal to the mesh size. Note however that in Figures 16 and 17, we have considered different mesh sizes $(25 \times 25,50 \times 50$ and $100 \times 100$ grids $)$, to measure the induced changes in the error estimates in the semiclassical limit.

\subsection{Nearly zero initial current}

We consider $L=0.5, a_{0}(x)=\exp \left(-80\left(\left(x_{1}-L / 2\right)^{2}+\left(x_{2}-L / 2\right)^{2}\right)\right)(1+\mathrm{i})$ and $\alpha=10^{-10}$ (hence $v_{\mid t=0}^{\varepsilon} \approx 0$ ).

Figure 1 shows the initial position and current densities. Figures 2 and 4 show the solutions at $T=0.1 \mathrm{~s}$ for $\varepsilon=0,0.001,0.01$ and 0.1 without and with the projection steps.

Figures 3 and 5 show the evolution of the constraints with time for different values of $\varepsilon$ without and with the projection steps. The original scheme can be seen being not conservative and dissipative. Of course, less dissipative numerical schemes could be used, but this does not remove the necessity for the projection step. Relative momentum constraint values appear being large, but one should keep in mind that these are in fact 

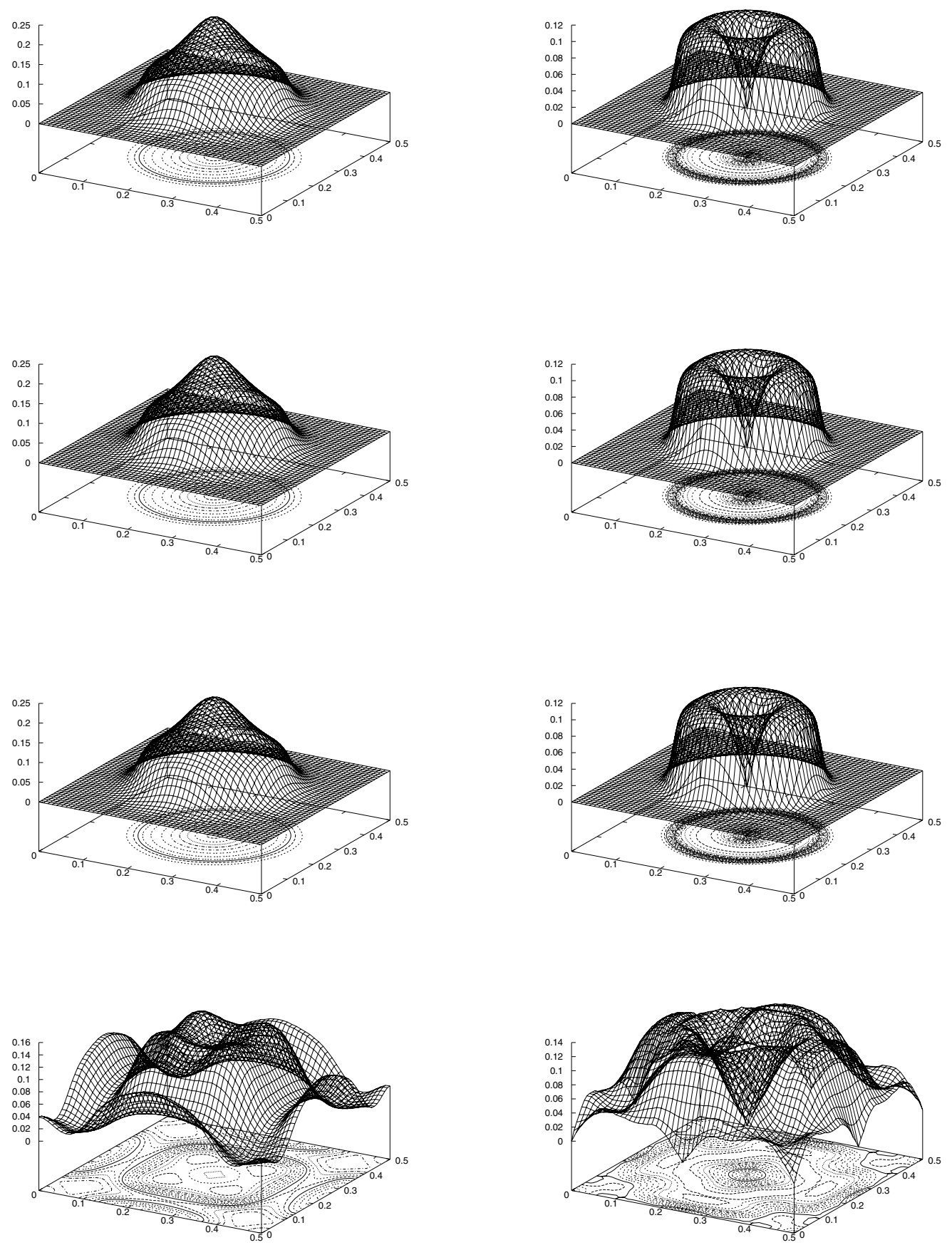

Figure 2. Without projection: evolution of the initial data from Figure 1. Position (left column) and norm of the current density vector (right column) at $T=0.1 \mathrm{~s}$ for (resp. from the top) $\varepsilon=0,0.001,0.01$ and 0.1 with $\alpha=10^{-10}$. 

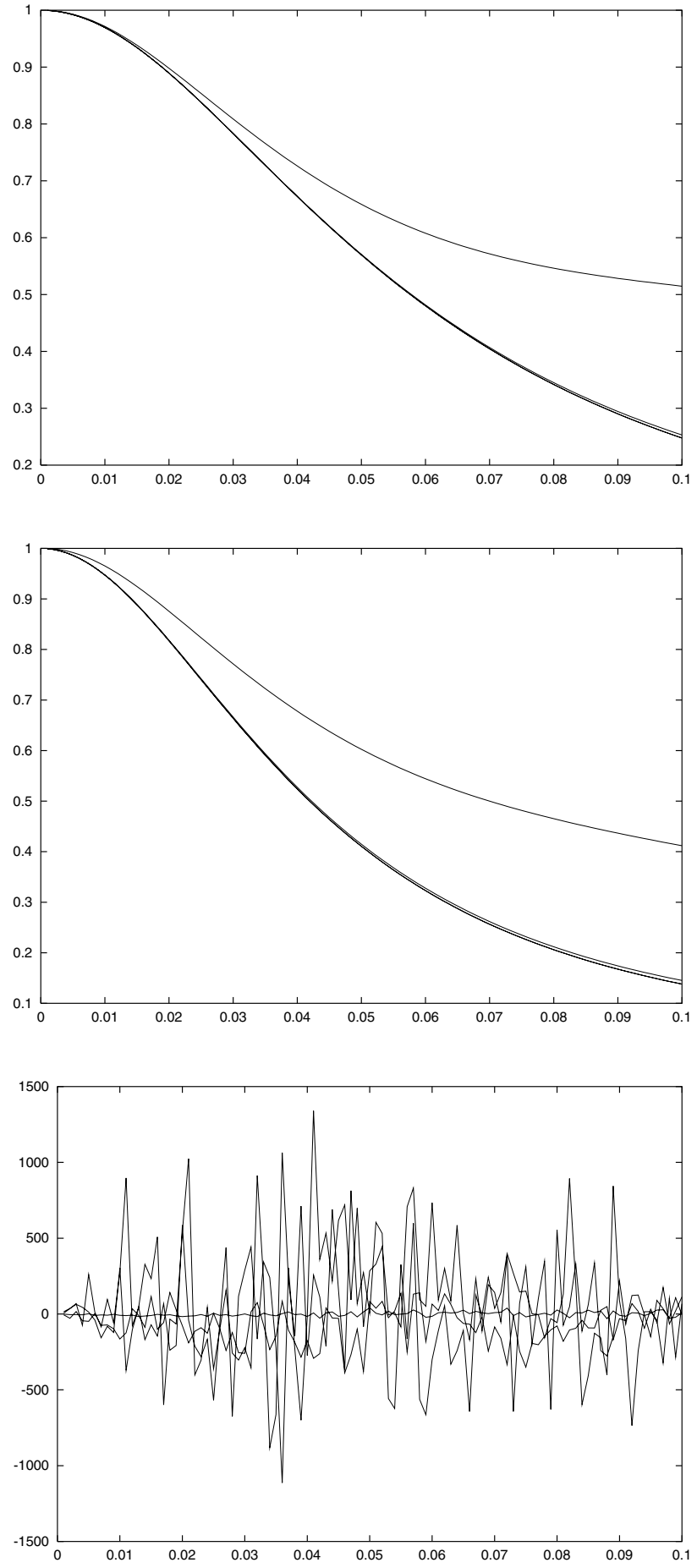

Figure 3. Without projection. Evolution in time (s) of (resp. from the top) the constraints on the position density, energy and sum of both components of the current density for $\varepsilon=$ $0,0.001,0.01$ and 0.1 for an initial condition with $\alpha=10^{-10}$. At the final time $T=0.1 \mathrm{~s}$, this corresponds to the solutions represented in Figure 2. 

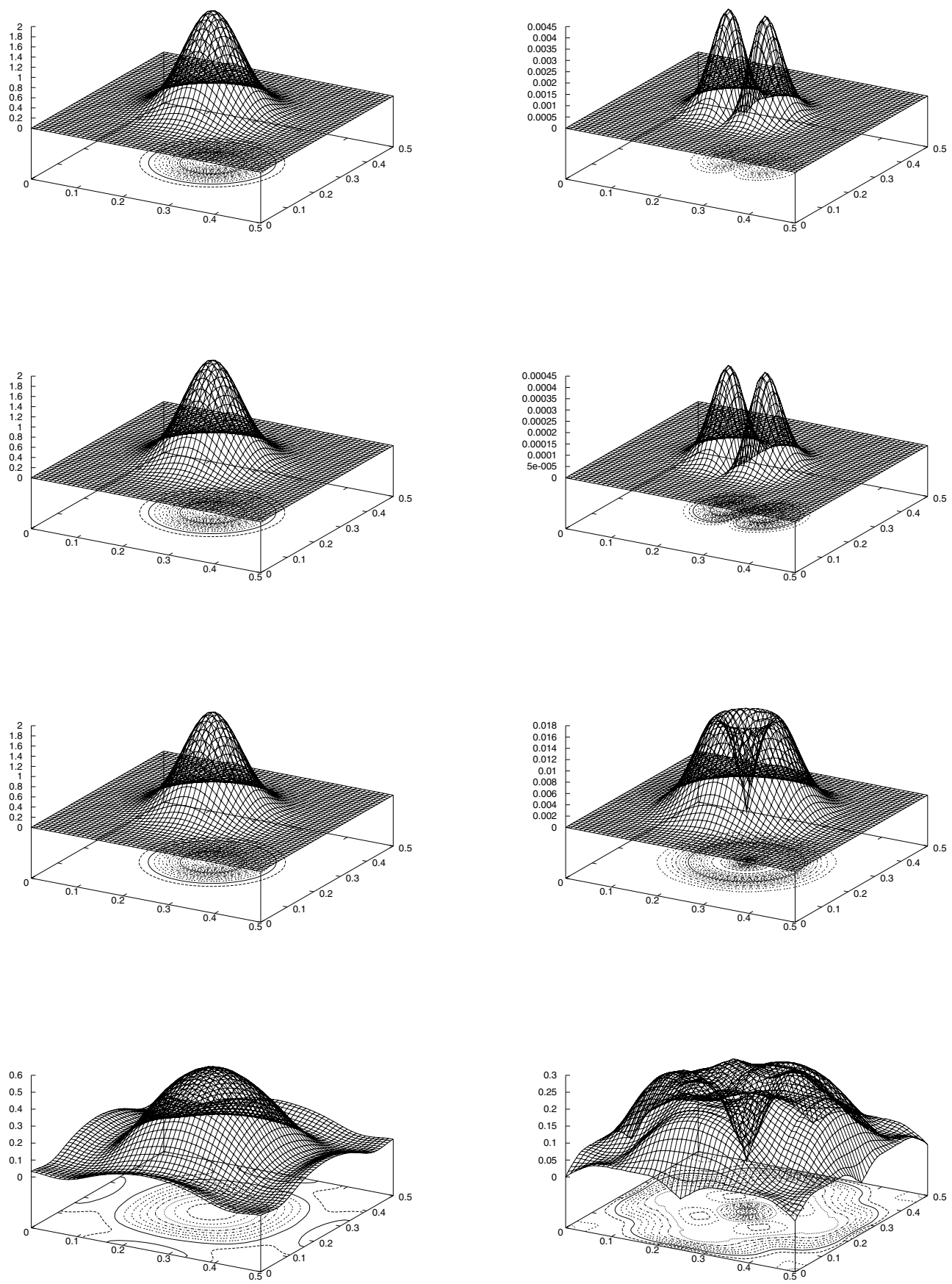

FIGURE 4. With projection: evolution of the initial data from Figure 1. Position (left column) and norm of the current density vector (right column) at $T=0.1 \mathrm{~s}$ for (resp. from the top) $\varepsilon=0,0.001,0.01$ and 0.1 with $\alpha=10^{-10}$. 

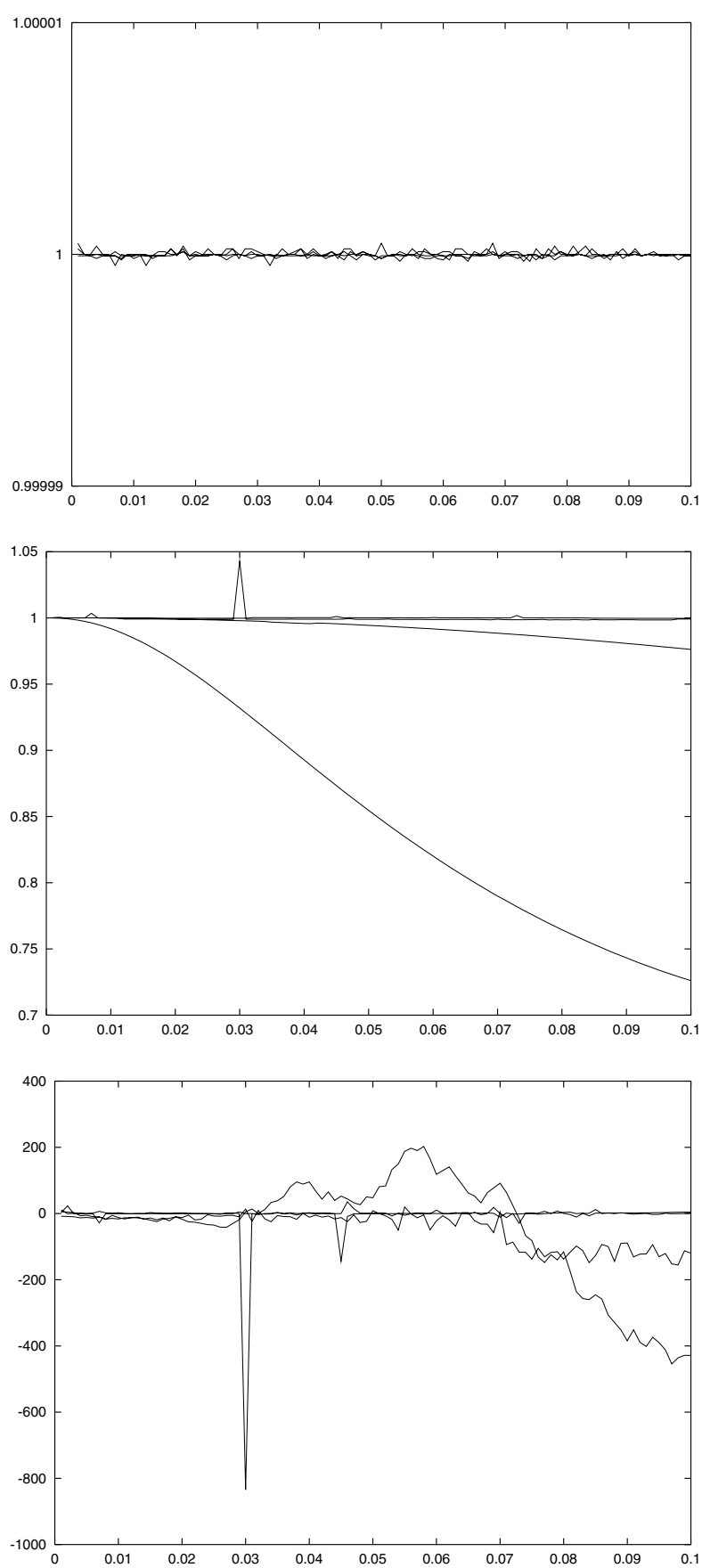

FIGURE 5. With projection. Evolution in time (s) of (resp. from the top) the constraints on the position density, energy and sum of both components of the current density for $\varepsilon=$ $0,0.001,0.01$ and 0.1 for an initial condition with $\alpha=10^{-10}$. At the final time $T=0.1 \mathrm{~s}$, this corresponds to the solutions represented in Figure 4. For the energy, deviation increases with $\varepsilon$. Larger oscillations appear with higher $\varepsilon$. Large values are due to the fact that initial value of the current is nearly zero. 


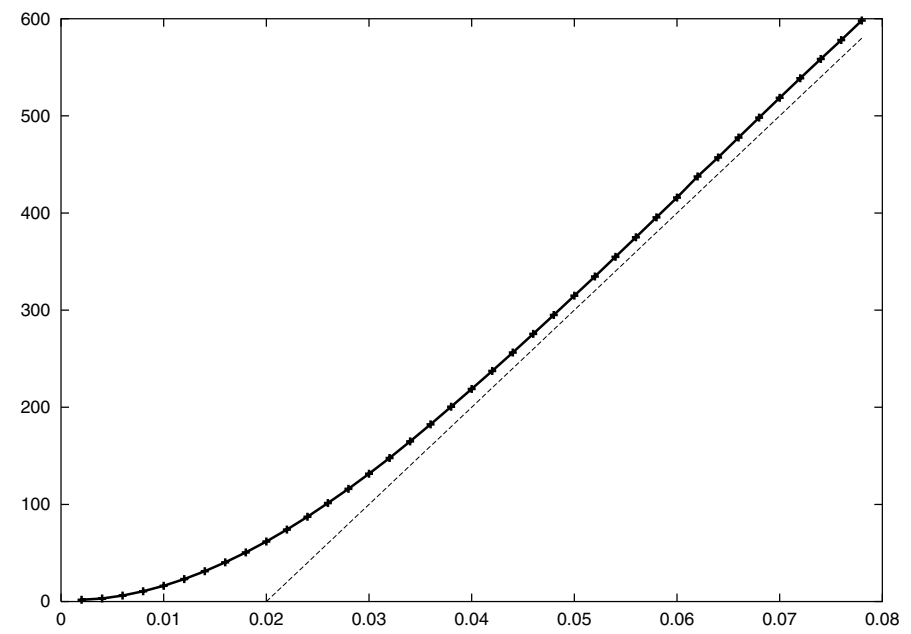

Figure 6. Linear dependency of $(4.1)$ at $T=0.1 \mathrm{~s}$ with respect to $\varepsilon$. This visualizes the evolution of the error in the semi-classical limit in Figure 4.

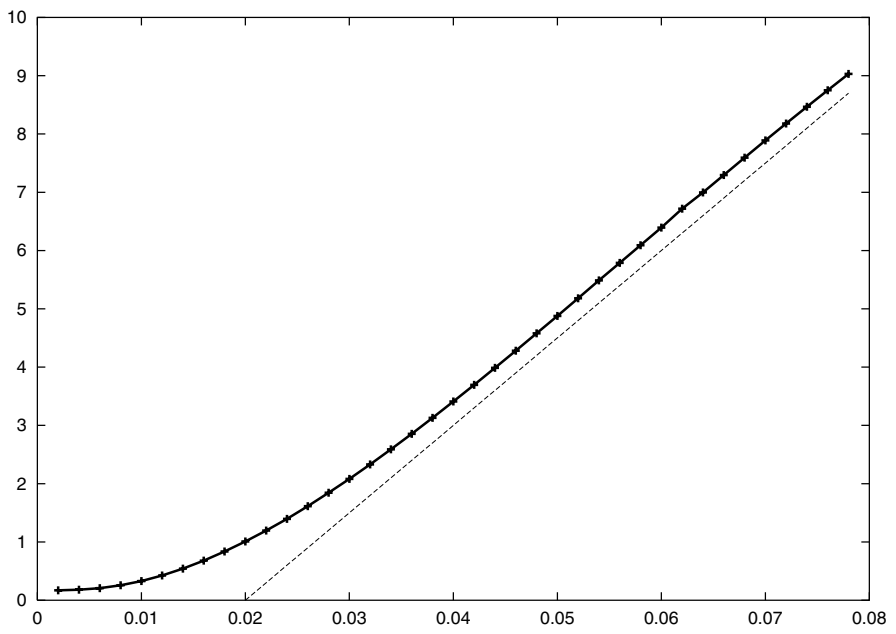

FigURE 7. Linear dependency of (4.2), which contains finer informations (such as "slow" oscillations) than (4.1), at $T=0.1 \mathrm{~s}$ with respect to $\varepsilon$.

very close to zero. What is most important is that mass and energy constraints cannot be satisfied at the same time. This can also be seen in the next case with initial current density.

An interesting indicator for the behavior of the solver is by checking if the following quantity is linear in $\varepsilon$ at a given time $T$ independent of $\varepsilon(\operatorname{see}(2.4))$ :

$$
\left\|\rho_{h, T}^{\varepsilon}-\rho_{h, T}\right\|_{L^{1}\left(\Omega_{h}\right)}+\left\|J_{h, T}^{\varepsilon}-J_{h, T}\right\|_{L^{1}\left(\Omega_{h}\right)} .
$$

This is shown in Figure 6 at $T=0.1 \mathrm{~s}$. The slope grows with time.

In the same way, Figure 7 shows the dependency with respect to $\varepsilon$ for the following quantity (see Thm. 2.1):

$$
\left\|a_{h, T}^{\varepsilon}-a_{h, T}\right\|_{L^{2}\left(\Omega_{h}\right)}+\left\|v_{h, T}^{\varepsilon}-v_{h, T}\right\|_{L^{2}\left(\Omega_{h}\right)} .
$$



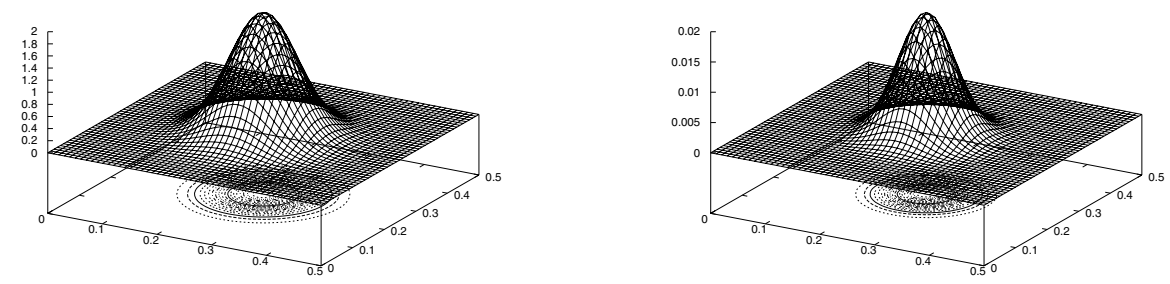

FiguRE 8. Initial position (left) and norm of the current density vector (right) with $\alpha=0.01$ (data considered in Sect. 4.2).

TABLE 1. The $L^{2}$ error (4.2) as a function of $\varepsilon$ and $h$, in the framework of Section 4.3.

\begin{tabular}{c|c|c|c}
\hline$\varepsilon$ & $h=1 / 50$ & $h=1 / 100$ & $h=1 / 200$ \\
\hline 0.078 & 4.913 & 11.035 & 22.458 \\
\hline 0.068 & 4.644 & 10.475 & 21.347 \\
\hline 0.058 & 4.297 & 9.747 & 19.888 \\
\hline 0.048 & 3.854 & 8.816 & 18.021 \\
\hline 0.038 & 3.296 & 7.641 & 15.665 \\
\hline 0.028 & 2.588 & 6.151 & 12.680 \\
\hline 0.018 & 1.658 & 4.189 & 8.744 \\
\hline 0.008 & 0.621 & 1.595 & 3.399 \\
\hline 0.002 & 0.099 & 0.203 & 0.418 \\
\hline
\end{tabular}

Again, the dependency is linear for small $\varepsilon$ at $T=0.1 \mathrm{~s}$.

For the three initial data studied in Section 4, (4.1) and (4.2) are assessed for 50 values of $\varepsilon$ (Figs. 6, 7, 11, 12, 16 and 17).

\subsection{Non zero initial current}

This is the same case as before but with $\alpha=10^{-2}$ and

$$
\left\{\begin{array}{l}
f(x)=\exp \left(-80\left(\left(x_{1}-L / 2\right)^{2}+\left(x_{2}-L / 2\right)^{2}\right)\right) \sin \left(10 x_{1}\right), \\
g(x)=\exp \left(-80\left(\left(x_{1}-L / 2\right)^{2}+\left(x_{2}-L / 2\right)^{2}\right)\right) \cos \left(10 x_{1}\right) .
\end{array}\right.
$$

Figure 8 shows the initial position and current densities. Figure 9 shows the solution at $T=0.1 \mathrm{~s}$ for $\varepsilon=0,0.001,0.01$ and 0.1 . Figure 10 shows the evolution of the position density, energy and current density constraints with time for different values of $\varepsilon$ when only mass through $I_{1}$ and the current density through vector $I_{3}$ have been maintained.

Figures 11 and 12 show that indicators (4.1) and (4.2) are still linear with respect to $\varepsilon$ but on a shorter range close to zero.

\section{3. $a^{\varepsilon}$ changing sign}

To introduce a changing sign initial data for $a^{\varepsilon}$, we consider an initial condition given by $a_{0}(x)=(\exp (-320$ $\left.\left.\left(\left(x_{1}-L / 2\right)^{2}+\left(x_{2}-L / 2\right)^{2}\right)\right)-\exp \left(-320\left(\left(x_{1}-L / 2\right)^{2}+\left(x_{2}-L / 2\right)^{2}\right)\right)\right)(1+\mathrm{i})$. This initial amplitude changes signs: the set where it is zero corresponds to the presence of vacuum in the hydrodynamical point of view. The initial current is as for the previous case with $f$ and $g$ given in (4.3). Figure 13 shows the initial position and 

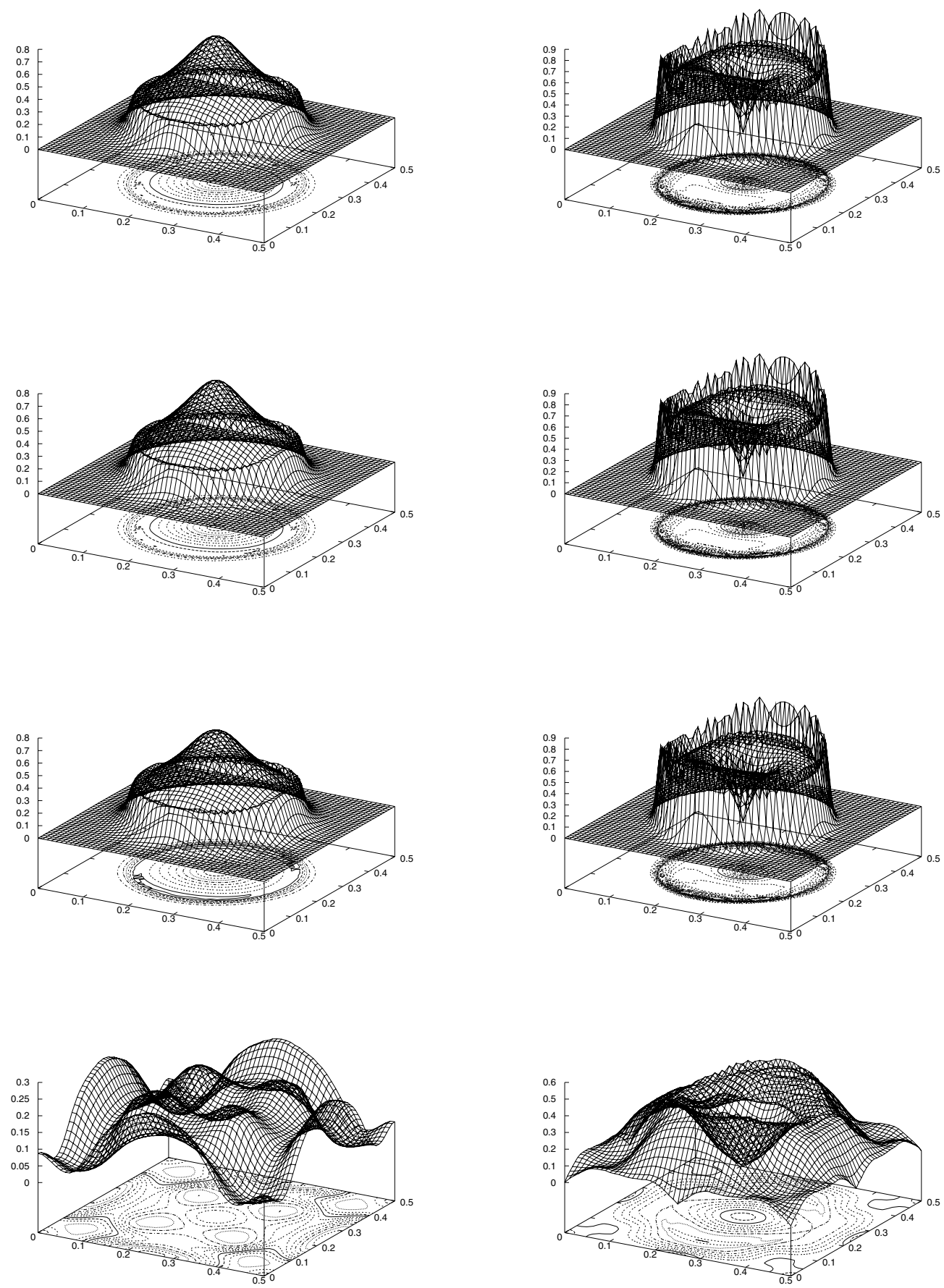

Figure 9. Evolution of the initial data from Figure 8 (with projection). Position (left column) and current density (right column) at $T=0.1 \mathrm{~s}$ for (resp. from the top) $\varepsilon=0,0.001,0.01$ and 0.1 with $\alpha=0.01$. 

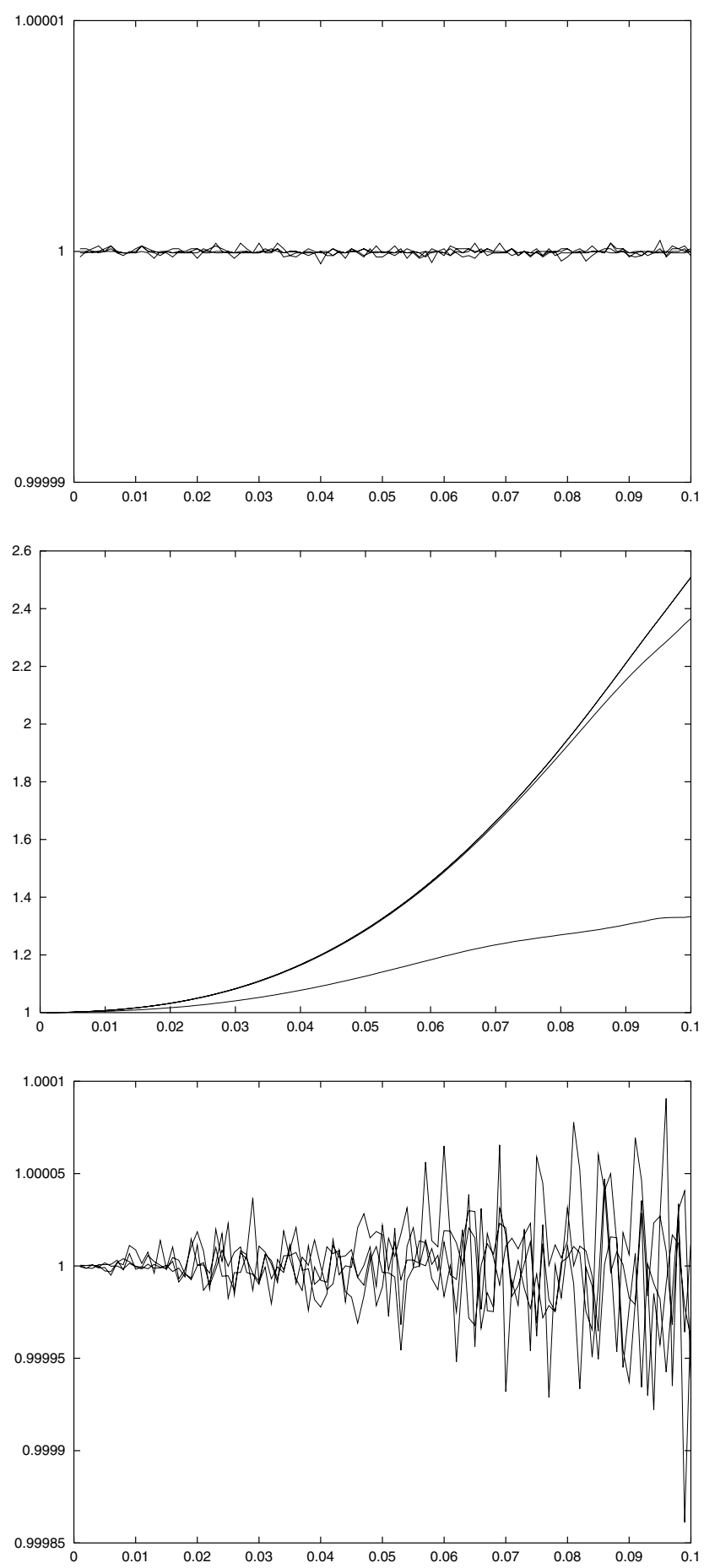

Figure 10. Evolution in time (s) of the constraints on the position density, energy and sum of both components of the current density (resp. from the top) for $\varepsilon=0,0.001,0.01$ and 0.1 for an initial condition with $\alpha=0.01$. At the final time $T=0.1 \mathrm{~s}$, this corresponds to the solutions represented in Figure 9. 


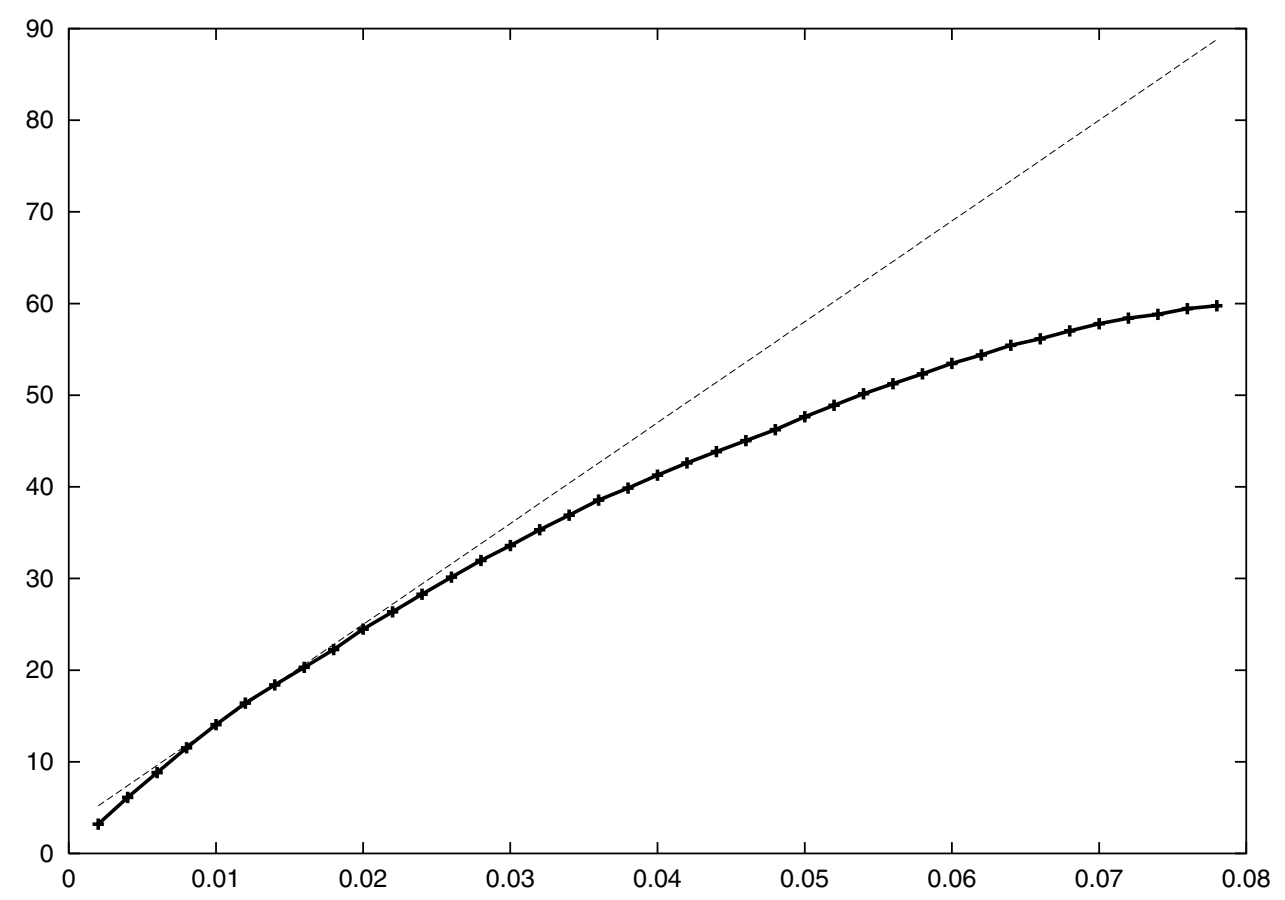

FiguRE 11. Linear dependency of (4.1) at $T=0.1 \mathrm{~s}$ with respect to $\varepsilon$, corresponding to Figure 9.

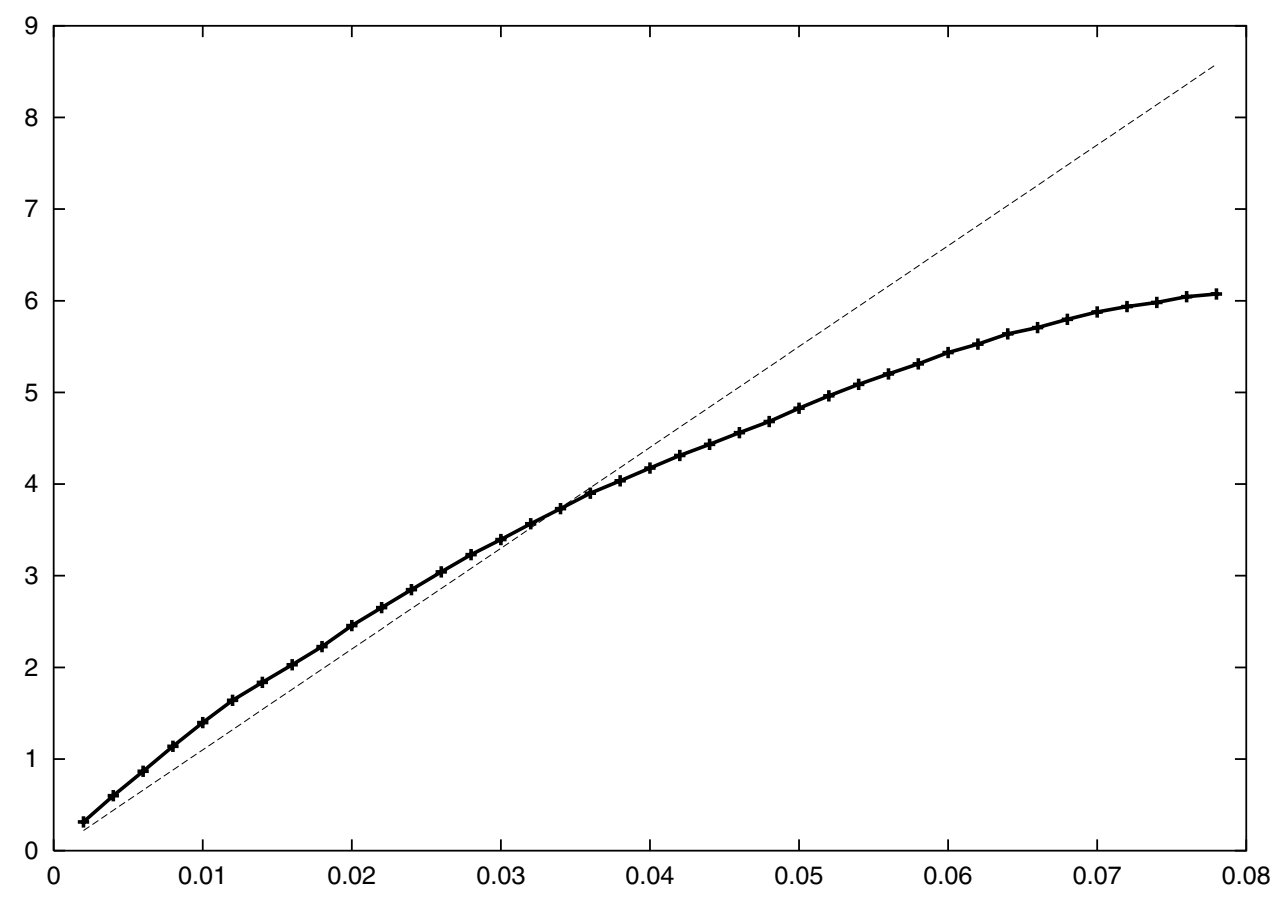

FiguRE 12. Linear dependency of $(4.2)$ at $T=0.1 \mathrm{~s}$ with respect to $\varepsilon$, corresponding to Figure 9. 

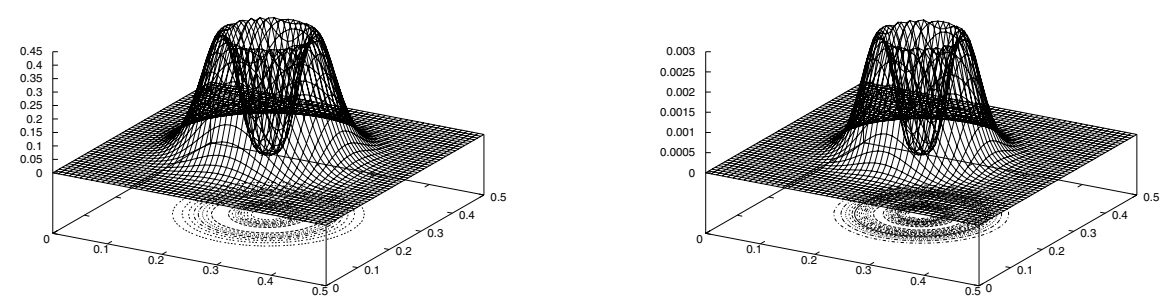

FIGURE 13. Initial position (left) and norm of the current density vector (right) with varying sign initial $a^{\varepsilon}$.

current densities. Figure 14 shows the solution at $T=0.05 \mathrm{~s}$ for $\varepsilon=0,0.001,0.01$ and 0.1 . Figure 15 shows the evolution of the position density, energy and current density constraints with time for different values of $\varepsilon$ when only mass through $I_{1}$ and the current density through vector $I_{3}$ have been maintained.

Figures 16 and 17 show indicators (4.1) and (4.2), for different values of the mesh size. In these figures, each curve corresponds to 50 numerical tests. We see that the error is indeed $\mathcal{O}(\varepsilon)$ in each case. However, it seems that the constant in the $\mathcal{O}(\varepsilon)$ depends on $h$. Even though for each $h$, we retrieve the $\mathcal{O}(\varepsilon)$ error in the semiclassical limit, as predicted by the theory, the corresponding constant is roughly doubled when $h$ is divided by two, in Figure 17. This may be easier to see on the corresponding table below, where we consider only nine of the fifty tests realized to obtain Figure 17.

To see the behavior of the approach after singularities have formed in the Euler equation (for $\varepsilon=0$ ), we show in Figure 18 the solution at $T=0.15 \mathrm{~s}$ : the solution for $\varepsilon=0$ has become singular, while the solution for $\varepsilon>0$ seems to remain smooth. In this case, the meaning of the figure for $\varepsilon=0$ is unclear, since we know that the scheme has dealt with a singularity. On the other hand, rapid oscillations have appeared at least for $\varepsilon=0.1$. For $\varepsilon=0.001$, the map is not very smooth, as if some oscillations were not resolved. Recall however that the time step and the mesh size are independent of $\varepsilon$ : in the presence of rapid oscillations, this strategy has proven unefficient in [6], as recalled in Section 1.2. This may very well be the case in Figure 18.

\section{Conclusion}

We have presented a numerical implementation to compute the solution of the system (1.7), which is a way to solve the nonlinear Schrödinger equation that yields correct (macroscopic) physical behavior, in the semiclassical limit, even when the numerical computation is underresolved (for mesh size and time step much larger than the Planck constant). To reconstruct the wave function $u^{\varepsilon}$, the phase $\phi^{\varepsilon}$ can be computed by a simple time integration, in view of (2.3).

The scheme used in this paper is explicit, and is therefore rather cheap on the computational level. It preserves the $L^{2}$-norm of the solution to the nonlinear Schrödinger equation, and can be adapted in order to conserve the momentum as well, thanks to simple projections based on rescaling. On the other hand, the energy is not conserved.

With mesh sizes and time steps which are independent of the Planck constant $\varepsilon$, we retrieve moreover the main two quadratic observables (position and current densities) in the semiclassical limit $\varepsilon \rightarrow 0$, and before singularities are formed in the limiting Euler equation, up to an error of order $\mathcal{O}(\varepsilon)$, as predicted by theoretical results. The presence of vacuum (zeroes of the position density) is not a problem in this approach; the case treated in Section 4.3 is in perfect agreement with this theoretical result.

Finally, these experiments suggest that once the solution to the Euler equation has developped singularities, the solution to (1.7) may remain smooth, while it becomes rapidly oscillatory. It is possibly $\varepsilon$-oscillatory in the sense of [23], but the existence of intermediary scales of oscillation cannot be a priori ruled out. We do not claim 

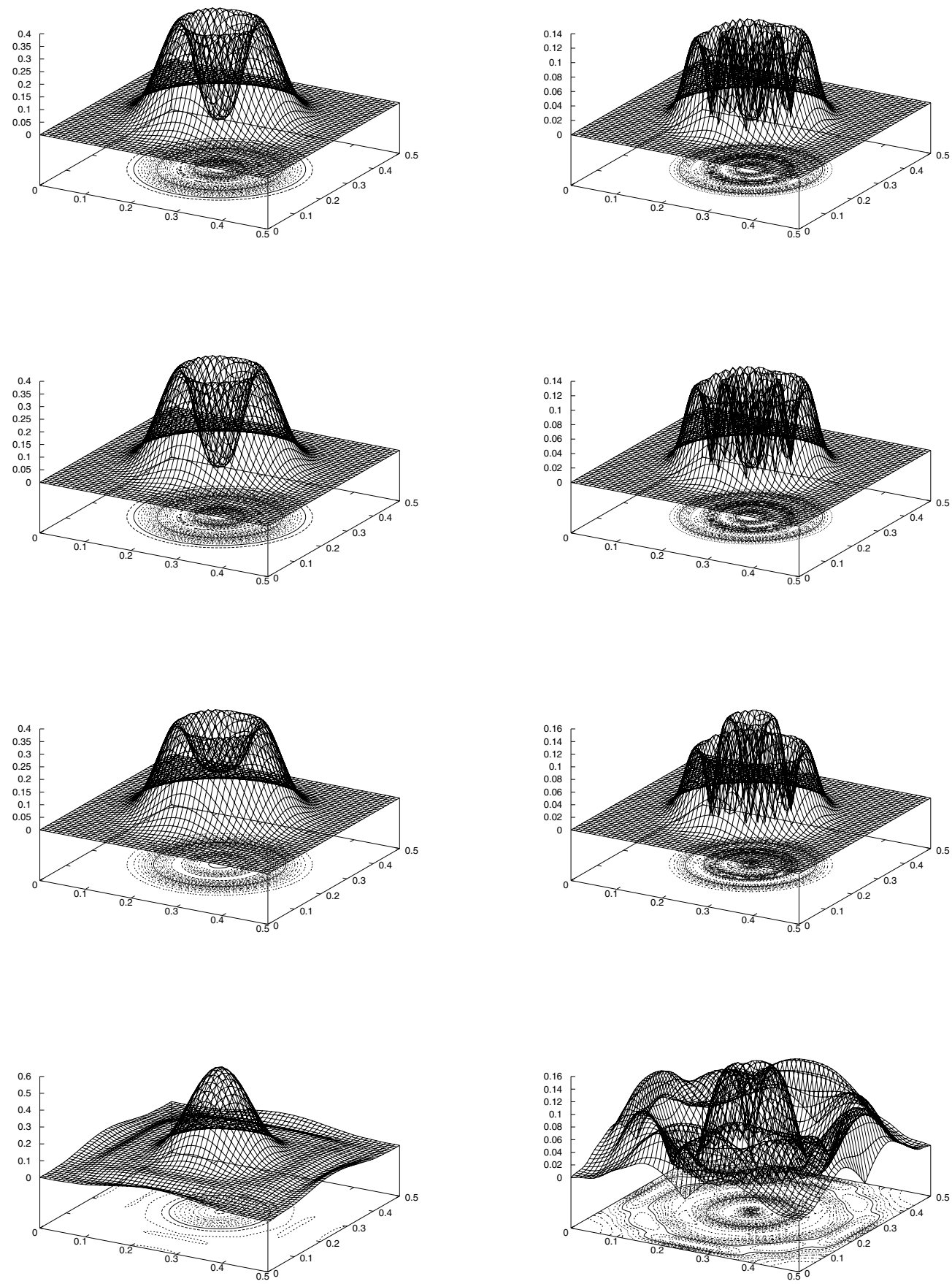

Figure 14. Evolution of the initial data from Figure 13. Position (left column) and current density (right column) at $T=0.05 \mathrm{~s}$ for (resp. from the top) $\varepsilon=0,0.001,0.01$ and 0.1 with $\alpha=0.01$. 

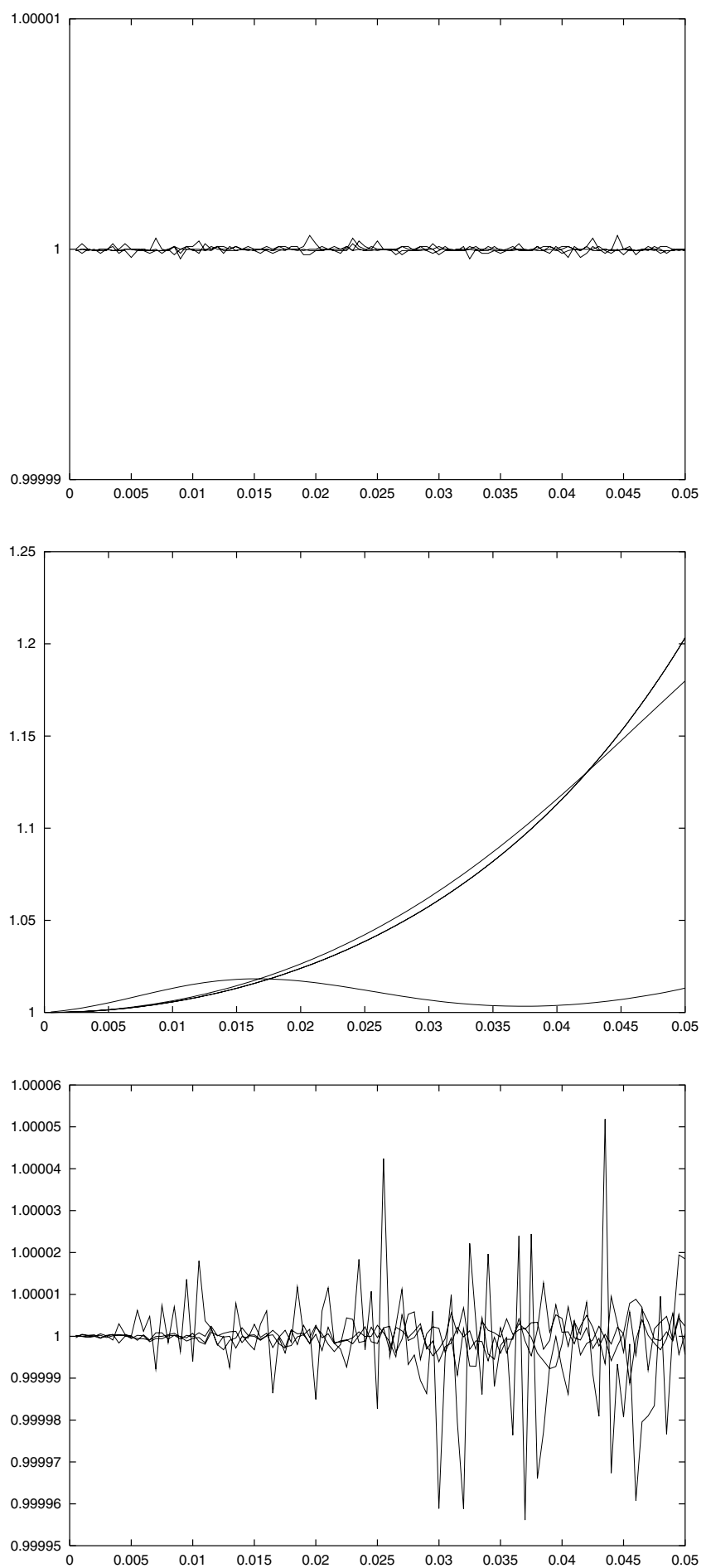

FIGURE 15. Evolution in time (s) of the constraints on the position density, energy and sum of both components of the current density (resp. from the top) for $\varepsilon=0,0.001,0.01$ and 0.1 for an initial condition having sign variation in $a^{\varepsilon}$. 


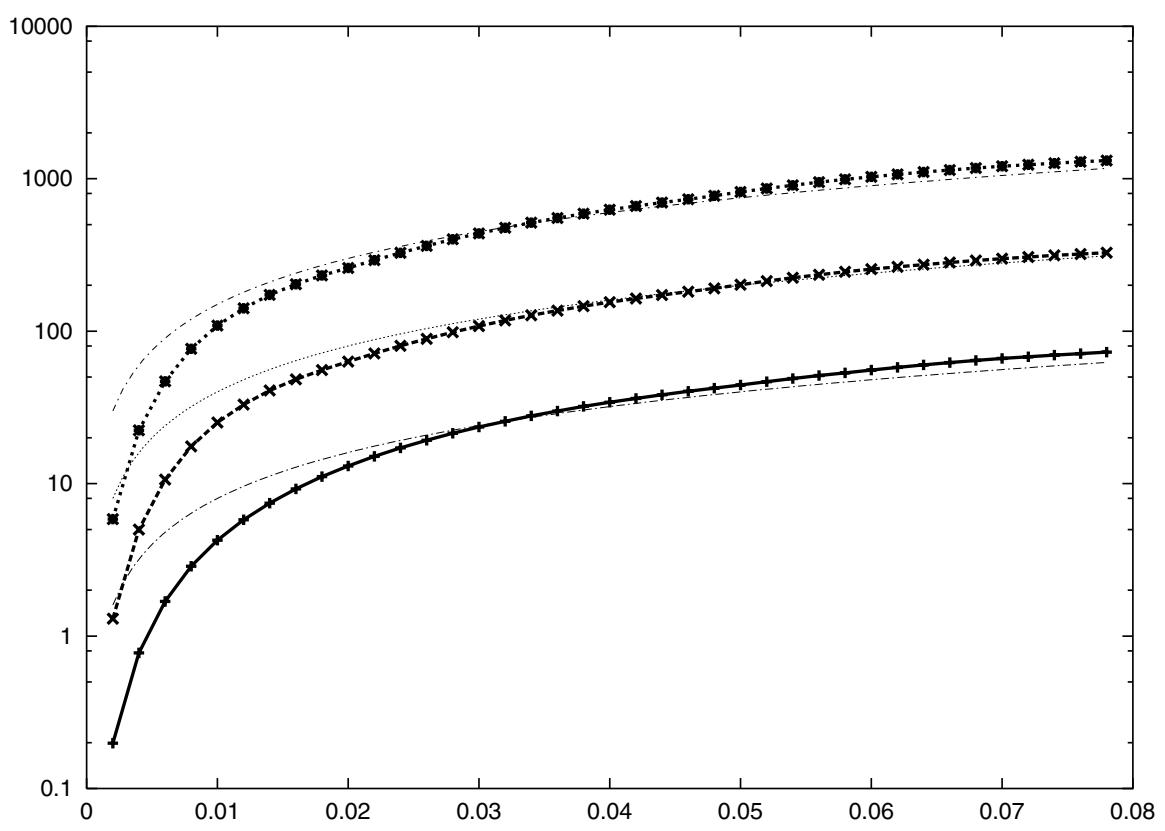

FiguRE 16. Linear dependency of (4.1) at $T=0.05 \mathrm{~s}$ with respect to $\varepsilon$, in logarithmic scale, with an initial condition having sign variation in $a^{\varepsilon}$, for three values of the mesh size $h$ : from bottom to top, $1 / h=50,100,200$. For $1 / h=100$, this corresponds to Figure 14 .

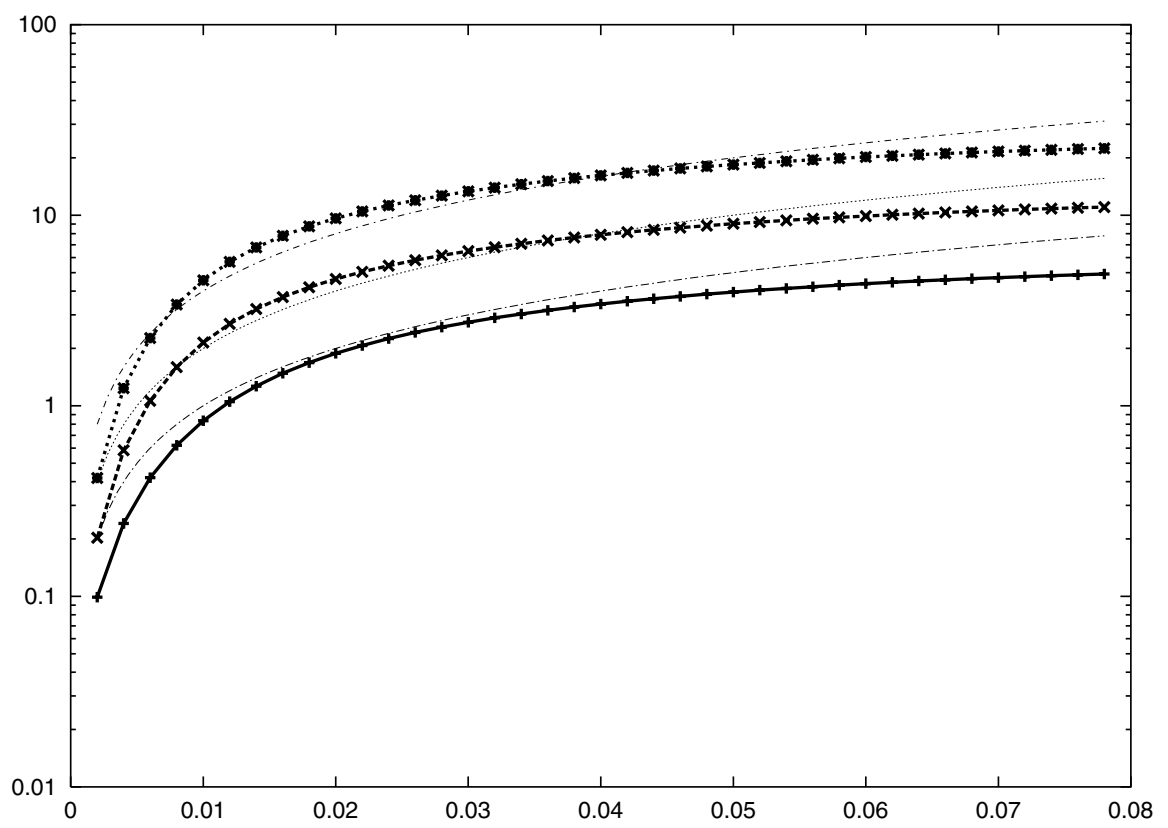

Figure 17. Same as in Figure 16, except that the $L^{2}$-norm (4.2) is measured, instead of the $L^{1}$-norm (4.1). 

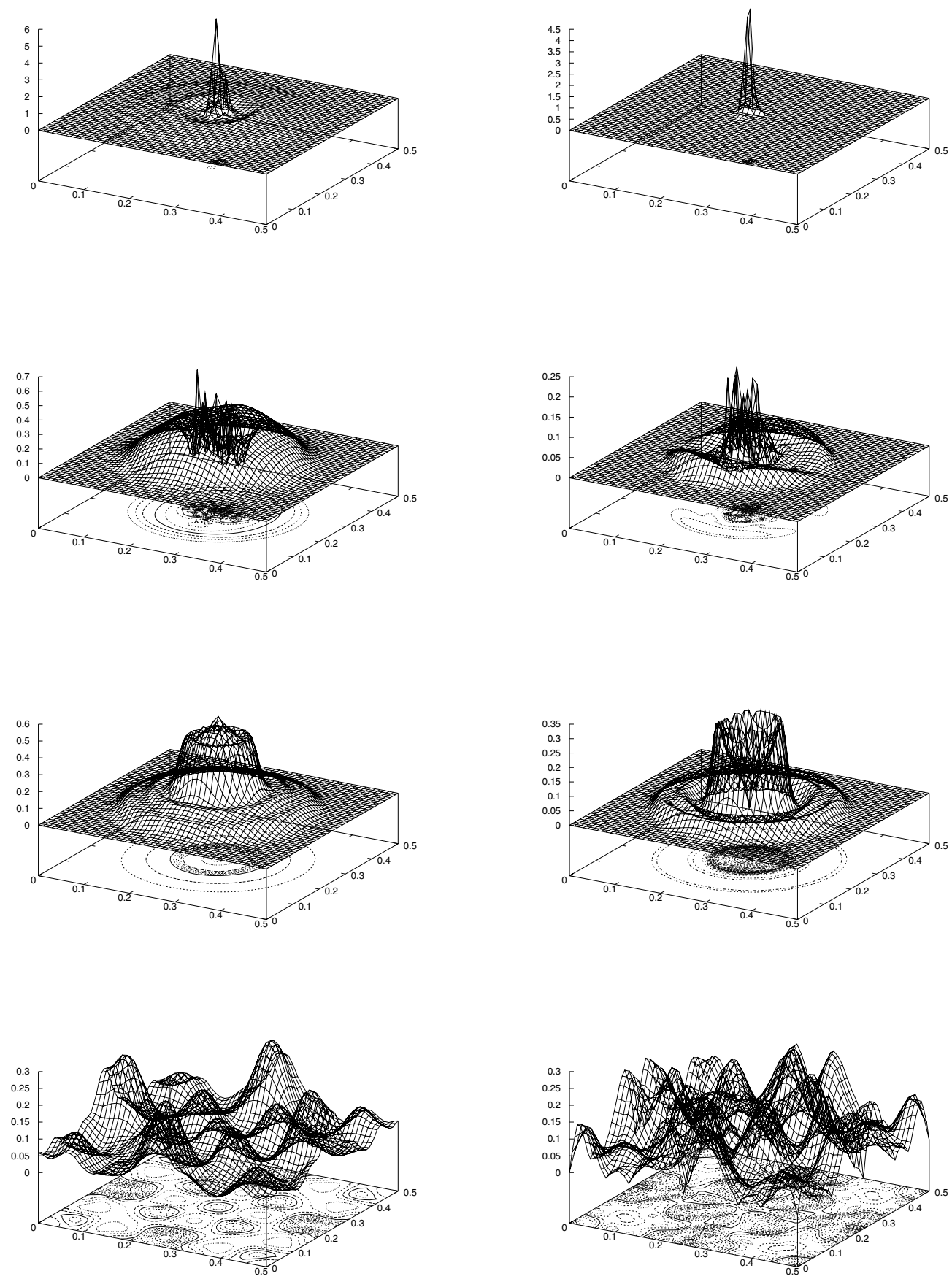

FiguRE 18. Evolution of the initial data from Figure 13 after the critical time for the Euler equation. Position (left column) and current density (right column) at $T=0.15 \mathrm{~s}$ for (resp. from the top) $\varepsilon=0,0.001,0.01$ and 0.1 nearly after the solution blows up for $\varepsilon=0$. 
to observe any quantitative result for post-breakup time, but rather a qualitative phenomenon: a refinement of time step and mesh size would be needed in view of a more reliable result after the breakup time. This aspect goes beyond the scope of the present paper.

Acknowledgements. The authors are grateful to the referees for their constructive comments.

\section{REFERENCES}

[1] F.Kh. Abdullaev, A. Gammal, L. Tomio and T. Frederico, Stability of trapped Bose-Einstein condensates. Phys. Rev. A 63 (2001) 043604.

[2] T. Alazard and R. Carles, Semi-classical limit of Schrödinger-Poisson equations in space dimension $n \geqslant 3 . J$. Diff. Eq. 233 (2007) $241-275$.

[3] T. Alazard and R. Carles, Supercritical geometric optics for nonlinear Schrödinger equations. Arch. Rational Mech. Anal. 194 (2009) 315-347.

[4] T. Alazard and R. Carles, WKB analysis for the Gross-Pitaevskii equation with non-trivial boundary conditions at infinity. Ann. Inst. Henri Poincaré, Anal. Non Linéaire 26 (2009) 959-977.

[5] W. Bao, S. Jin and P.A. Markowich, On time-splitting spectral approximations for the Schrödinger equation in the semiclassical regime. J. Comput. Phys. 175 (2002) 487-524.

[6] W. Bao, S. Jin and P.A. Markowich, Numerical study of time-splitting spectral discretizations of nonlinear Schrödinger equations in the semiclassical regimes. SIAM J. Sci. Comput. 25 (2003) 27-64.

[7] C. Besse, A relaxation scheme for the nonlinear Schrödinger equation. SIAM J. Numer. Anal. 42 (2004) 934-952.

[8] C. Besse, B. Bidégaray and S. Descombes, Order estimates in time of splitting methods for the nonlinear Schrödinger equation. SIAM J. Numer. Anal. 40 (2002) 26-40.

[9] Y. Brenier and L. Corrias, A kinetic formulation for multi-branch entropy solutions of scalar conservation laws. Ann. Inst. Henri Poincaré, Anal. Non Linéaire 15 (1998) 169-190.

[10] R. Carles, Geometric optics and instability for semi-classical Schrödinger equations. Arch. Rational Mech. Anal. 183 (2007) 525-553.

[11] R. Carles, Semi-classical analysis for nonlinear Schrödinger equations. World Scientific Publishing Co. Pte. Ltd., Hackensack, NJ (2008).

[12] R. Carles and L. Gosse, Numerical aspects of nonlinear Schrödinger equations in the presence of caustics. Math. Models Methods Appl. Sci. 17 (2007) 1531-1553.

[13] T. Cazenave, Semilinear Schrödinger equations, Courant Lecture Notes in Mathematics 10. New York University Courant Institute of Mathematical Sciences, New York (2003).

[14] J.-Y. Chemin, Dynamique des gaz à masse totale finie. Asymptotic Anal. 3 (1990) 215-220.

[15] D. Chiron and F. Rousset, Geometric optics and boundary layers for nonlinear Schrödinger equations. Comm. Math. Phys. 288 (2009) 503-546.

[16] F. Dalfovo, S. Giorgini, L.P. Pitaevskii and S. Stringari, Theory of Bose-Einstein condensation in trapped gases. Rev. Mod. Phys. 71 (1999) 463-512.

[17] P. Degond, S. Gallego and F. Méhats, An asymptotic preserving scheme for the Schrödinger equation in the semiclassical limit. C.R. Math. Acad. Sci. Paris 345 (2007) 531-536.

[18] P. Degond, S. Jin and M. Tang, On the time splitting spectral method for the complex Ginzburg-Landau equation in the large time and space scale limit. SIAM J. Sci. Comput. 30 (2008) 2466-2487.

[19] J.J. Duistermaat, Oscillatory integrals, Lagrange immersions and unfolding of singularities. Comm. Pure Appl. Math. 27 (1974) 207-281.

[20] A. Gammal, T. Frederico, L. Tomio and Ph. Chomaz, Atomic Bose-Einstein condensation with three-body intercations and collective excitations. J. Phys. B 33 (2000) 4053-4067.

[21] C.L. Gardner, The quantum hydrodynamic model for semiconductor devices. SIAM J. Appl. Math. 54 (1994) $409-427$.

[22] P. Gérard, Remarques sur l'analyse semi-classique de l'équation de Schrödinger non linéaire, Séminaire sur les Équations aux Dérivées Partielles, 1992-1993. École Polytech., Palaiseau (1993), http://www.numdam.org/numdam-bin/fitem?id=SEDP_ 1992-1993__A13_0www.numdam.org, pp. Exp. No. XIII, 13.

[23] P. Gérard, P.A. Markowich, N.J. Mauser and F. Poupaud, Homogenization limits and Wigner transforms. Comm. Pure Appl. Math. 50 (1997) 323-379.

[24] J. Ginibre and G. Velo, On a class of nonlinear Schrödinger equations. I The Cauchy problem, general case. J. Funct. Anal. 32 (1979) 1-32

[25] L. Gosse, Using K-branch entropy solutions for multivalued geometric optics computations. J. Comput. Phys. 180 (2002) $155-182$ 
[26] L. Gosse, A case study on the reliability of multiphase WKB approximation for the one-dimensional Schrödinger equation, Numerical methods for hyperbolic and kinetic problems, IRMA Lect. Math. Theor. Phys. 7. Eur. Math. Soc., Zürich (2005) $131-141$.

[27] E. Grenier, Semiclassical limit of the nonlinear Schrödinger equation in small time. Proc. Amer. Math. Soc. 126 (1998) 523-530.

[28] S. Jin, Efficient asymptotic-preserving (AP) schemes for some multiscale kinetic equations. SIAM J. Sci. Comput. 21 (1999) 441-454.

[29] C. Josserand and Y. Pomeau, Nonlinear aspects of the theory of Bose-Einstein condensates. Nonlinearity 14 (2001) R25-R62.

[30] H. Li and C.-K. Lin, Semiclassical limit and well-posedness of nonlinear Schrödinger-Poisson systems. Electron. J. Diff. Eq. (2003) 17 (electronic).

[31] H. Liu and E. Tadmor, Semiclassical limit of the nonlinear Schrödinger-Poisson equation with subcritical initial data. Methods Appl. Anal. 9 (2002) 517-531.

[32] E. Madelung, Quanten theorie in Hydrodynamischer Form. Zeit. Physik 40 (1927) 322.

[33] T. Makino, S. Ukai and S. Kawashima, Sur la solution à support compact de l'équation d'Euler compressible. Japan J. Appl. Math. 3 (1986) 249-257.

[34] P.A. Markowich, P. Pietra and C. Pohl, Numerical approximation of quadratic observables of Schrödinger-type equations in the semi-classical limit. Numer. Math. 81 (1999) 595-630.

[35] S. Masaki, Local existence and WKB approximation of solutions to Schrödinger-Poisson system in the two-dimensional whole space. Comm. Partial Differential Equations 35 (2010) 2253-2278.

[36] V.P. Maslov and M.V. Fedoriuk, Semiclassical approximation in quantum mechanics, Mathematical Physics and Applied Mathematics 7. Translated from the Russian by J. Niederle and J. Tolar, Contemporary Mathematics 5. D. Reidel Publishing Co., Dordrecht (1981).

[37] G. Métivier, Remarks on the well-posedness of the nonlinear Cauchy problem, Geometric analysis of PDE and several complex variables, Contemp. Math. 368. Amer. Math. Soc., Providence, RI (2005) 337-356.

[38] H. Michinel, J. Campo-Táboas, R. García-Fernández, J.R. Salgueiro and M.L. Quiroga-Teixeiro, Liquid light condensates. Phys. Rev. E 65 (2002) 066604.

[39] B. Mohammadi and J.H. Saiac, Pratique de la simulation numérique. Dunod, Paris (2003).

[40] J. Nocedal and S.J. Wright, Numerical optimization. 2d edition, Springer Series in Operations Research and Financial Engineering, Springer, New York (2006).

[41] L. Pitaevskii and S. Stringari, Bose-Einstein condensation, International Series of Monographs on Physics 116. The Clarendon Press Oxford University Press, Oxford (2003).

[42] E. Ryckman and M. Visan, Global well-posedness and scattering for the defocusing energy-critical nonlinear Schrödinger equation in $\mathbb{R}^{1+4}$. Amer. J. Math. 129 (2007) 1-60.

[43] G. Strang, Introduction to applied mathematics. Applied Mathematical Sciences, Wellesley-Cambridge Press, New York (1986).

[44] C. Sulem and P.-L. Sulem, The nonlinear Schrödinger equation, self-focusing and wave collapse. Springer-Verlag, New York (1999).

[45] M. Taylor, Partial differential equations. III, Applied Mathematical Sciences 117. Nonlinear equations. Springer-Verlag, New York (1997).

[46] L. Thomann, Instabilities for supercritical Schrödinger equations in analytic manifolds. J. Diff. Eq. 245 (2008) $249-280$.

[47] Z. Xin, Blowup of smooth solutions of the compressible Navier-Stokes equation with compact density. Comm. Pure Appl. Math. 51 (1998) 229-240.

[48] V.E. Zakharov and S.V. Manakov, On the complete integrability of a nonlinear Schrödinger equation. Theor. Math. Phys. 19 (1974) 551-559.

[49] V.E. Zakharov and A.B. Shabat, Interaction between solitons in a stable medium. Sov. Phys. JETP 37 (1973) $823-828$. 